\title{
Functional bottlenecks for generation of HIV-1 intersubtype Env recombinants
}

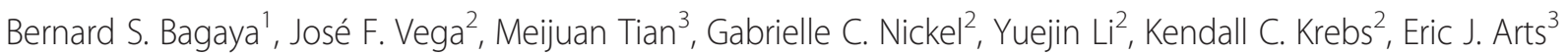
and Yong Gao ${ }^{1,2^{*}}$

\begin{abstract}
Background: Intersubtype recombination is a powerful driving force for HIV evolution, impacting both HIV-1 diversity within an infected individual and within the global epidemic. This study examines if viral protein function/ fitness is the major constraint shaping selection of recombination hotspots in replication-competent HIV-1 progeny. A better understanding of the interplay between viral protein structure-function and recombination may provide insights into both vaccine design and drug development.

Results: In vitro HIV-1 dual infections were used to recombine subtypes A and D isolates and examine breakpoints in the Env glycoproteins. The entire env genes of 21 A/D recombinants with breakpoints in gp120 were non-functional when cloned into the laboratory strain, NL4-3. Likewise, cloning of A/D gp120 coding regions also produced dead viruses with non-functional Envs. 4/9 replication-competent viruses with functional Env's were obtained when just the V1-V5 regions of these same A/D recombinants (i.e. same A/D breakpoints as above) were cloned into NL4-3.

Conclusion: These findings on functional A/D Env recombinants combined with structural models of Env suggest a conserved interplay between the $\mathrm{C} 1$ domain with $\mathrm{C} 5$ domain of gp120 and extracellular domain of gp41. Models also reveal a co-evolution within $\mathrm{C1}, \mathrm{C5}$, and ecto-gp41 domains which might explain the paucity of intersubtype recombination in the gp120 V1-V5 regions, despite their hypervariability. At least HIV-1 A/D intersubtype recombination in gp120 may result in a C1 from one subtype incompatible with a C5/gp41 from another subtype.
\end{abstract}

\section{Background}

A major obstacle for HIV treatment and vaccine development is virus diversity which continues to increase due to its high mutation rate and recombination [1-6]. Intersubtype recombination is shaping HIV evolution by establishing unique and stable circulating recombinant forms (URFs and CRFs) in various regional epidemics [7-14], by contributing to the rapid emergence of multidrug resistance $[15,16]$ and immune escape $[17,18]$, and by rescuing HIV-1 from catastrophic mutations via negative epistasis [19]. In this study, we have explored the functional constraints that limit intersubtype recombination in the HIV-1 env gene. These mechanistic studies on HIV-1 recombination can provide valuable insight

\footnotetext{
* Correspondence: yxg18@case.edu

'Department of Molecular Biology and Microbiology, School of Medicine, Case Western Reserve University, 10900 Euclid Ave, Cleveland, OH 44106, USA

${ }^{2}$ Division of Infectious Diseases, Department of Medicine, Case Western Reserve University, 10900 Euclid Ave, Cleveland, OH 44106, USA Full list of author information is available at the end of the article
}

into chimeric env cloning and production, the basis for many HIV-1 vaccine designs. Likewise, understanding the limitations in functional complementation within the env coding region can be advantageous as a therapeutic target and for drug design.

The HIV-1 envelope is a glycoprotein trimeric complex found on the viral surface, embedded in the membrane, and composed of the gp120 subunit spikes in a non-covalent interaction with the gp41 harboring the transmembrane domain. Each gp120-gp41 subunit is derived from the proteolytic processing of the envelope precursor gp160 in the Golgi complex [20,21]. The envelope trimer collectively coordinates entry of HIV-1 into susceptible cells. The gp120 glycoprotein is subdivided into a conserved core derived from five conserved subdomains $(\mathrm{C} 1-\mathrm{C} 5)$ interspersed by five hypervariable, glycosylated loops (V1-V5) [22, 23]. The C4 region of gp120 mediates binding to the host CD4 molecule, inducing a conformational change, and promoting interaction between gp120 C2 and V3 regions with the $\mathrm{N}$

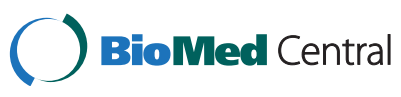

(C) 2015 Bagaya et al.; licensee BioMed Central. This is an Open Access article distributed under the terms of the Creative Commons Attribution License (http://creativecommons.org/licenses/by/4.0), which permits unrestricted use, distribution, and reproduction in any medium, provided the original work is properly credited. The Creative Commons Public Domain Dedication waiver (http://creativecommons.org/publicdomain/zero/1.0/) applies to the data made available in this article, unless otherwise stated. 
terminus and $2^{\text {nd }}$ extracellular loop of CCR5 (or CXCR4). The gp41 senses the conformational changes in gp120 and undergoes a radical structural refolding culminating in the fusion of viral and host cell membranes [24, 25]. The second exons of tat and rev overlap with the gp41 coding region of HIV-1 env, and must be correctly spliced to join the first exons to produce functional Tat and Rev proteins which are two essential viral regulatory factors for HIV gene expression [26, 27]. Thus, any modifications at the gp120/gp41 coding interface due to the intersubtype recombination, could alter the correct splicing of the tat and rev mRNA and possibly disrupt the function of Tat and Rev proteins. However, significant intersubtype sequence variability in both tat and rev sequences exists, and even in the same subtype Tat and Rev continue to evolve under selection pressure $[28,29]$.

We have previously examined the emergence and selection of intersubtype HIV-1 recombinants in single cycle systems involving replication defective viruses and in dual infection studies [1, 2, 30,31]. With increasing selection for replication competent viruses using various in vitro systems, we observed a re-distribution of env recombination sites within the gp120 coding sequence (no selection) to breakpoints primarily located in the gp120/gp41 interface (selection for fully functional Env's). Intersubtype recombination within the HIV-1 gp120 coding region could impact complementation between the subdomains of gp120 and produce Env glycoproteins that are not properly expressed, modified, or transported to the cell surface. Intersubtype recombinants with breakpoints in gp120 also provide a unique circumstance to study intermolecular interactions within the HIV-1 envelope. Even when incorporated into a new virus particle, such chimeric Envs may be defective for subsequent host cell entry. Likewise, a recombination breakpoint in the gp120 coding region of env could also impact the function of the accessory proteins, Rev and Tat. We have characterized a set of HIV-1 intersubtype A and D Env recombinants with breakpoints in the gp120 and gp41 coding region and have shown that A/D breakpoints between the C1 and C5 domains of gp120 result in non-functional, replicationdefective Env glycoproteins. This study now provides strong evidence of co-evolution and direct interplay between the C1, C5, and gp41 domains of the Env glycoproteins, necessary for host cell entry and viral infectivity.

\section{Results}

\section{Generation of Intersubtype envA/D recombinants}

We have previously described dual infection methods to produce HIV-1 env recombinants [32]. Even though PBMCs are more relevant in generating recombinants similar in HIV patients, based on our previous experience, the infection efficiency of HIV in PBMCs is much lower than in U87.CD4.CCR5 cell line resulting in very low levels of recombinant genomes, and the sites of intersubtype recombination was similar in U87 cells and PBMCs [1, 2, 30, 31]. In this study, subtype A (A91 and A115) and D (D109) HIV-1 isolates were used to co-infect U87.CD4.CCR5 cells. Subtypes A and D co-circulate in Kenya and Uganda and frequently recombine to generate A/D URFs, isolated in HIV-infected individuals. We cloned and sequenced 88 envA/D recombinants from dual infections of the A91 + D109 and of the A115+D109 HIV-1 isolates. Of 88 env $A / D$ recombinants, 28 had recombination breakpoints within gp120 while the remaining breakpoints mapped to the highly conserved, transmembrane coding region of gp 41 . Seven A/D gp120 recombinants were excluded based on premature stop codons or frameshift in the envelope open reading frame (ORF) or duplicates with the same unique breakpoint. The remaining 21 unique A/D recombinants with breakpoints in gp120, 11 A91/D109 (Fig. 1a) and $10 \mathrm{~A} 115 / \mathrm{D} 109$ (Fig. 1b), were used to investigate the impact of gp120 recombination on Env function. Except bk6780 (breakpoint at position 6780) and bk7643, all of the other breakpoints identified in the gp120 region were single (Fig. 1).

\section{Full length HIV-1 A/D recombinant envelopes with breakpoints in gp120 were non-functional when cloned into NL4-3 backbone}

We and others have previously described intersubtype recombination "hotspots" in the env gene derived from single-cycle assays, dual infection systems, and recombination enrichment systems involving siRNA selection [1, 2, 30-33]. With increased selection for replicationcompetent viruses, there was limited intersubtype recombination within the gp120 coding regions and increased detection of breakpoints in the gp41 coding regions. In this study, we examined the function of Env when intersubtype recombination was identified in the gp120 coding sequence. We first cloned full length env with A/D gp120 breakpoints (aka env-gp120 A/D) into pREC_nfl_HIV $\Delta$ env/URA3 plasmid (containing an HIV1 NL4-3 near full length genome except 5'LTR sequence as a backbone) (Fig. 2a) using a yeast-based recombination method [34]. The cloned env-gp120 A/D recombinants in the NL4-3 backbone were tested for their ability to mediate cell fusion using the Veritrop assay (a surrogate of virus entry) as previously described [35]. These env-gp120 A/D vectors were also co-transfected with a complementing vector [34] to measure virus production and subsequent multiple cycle replication.

All of the parental A91, A115 and D109 HIV-1 env genes in pREC_nfl_HIV $\mathrm{NL4-3}_{3}$ mediated robust cell-to-cell fusion when tested by using the Veritrop assay [35] (Fig. 3a). However, the 21 env-gp120 A/D recombinants 


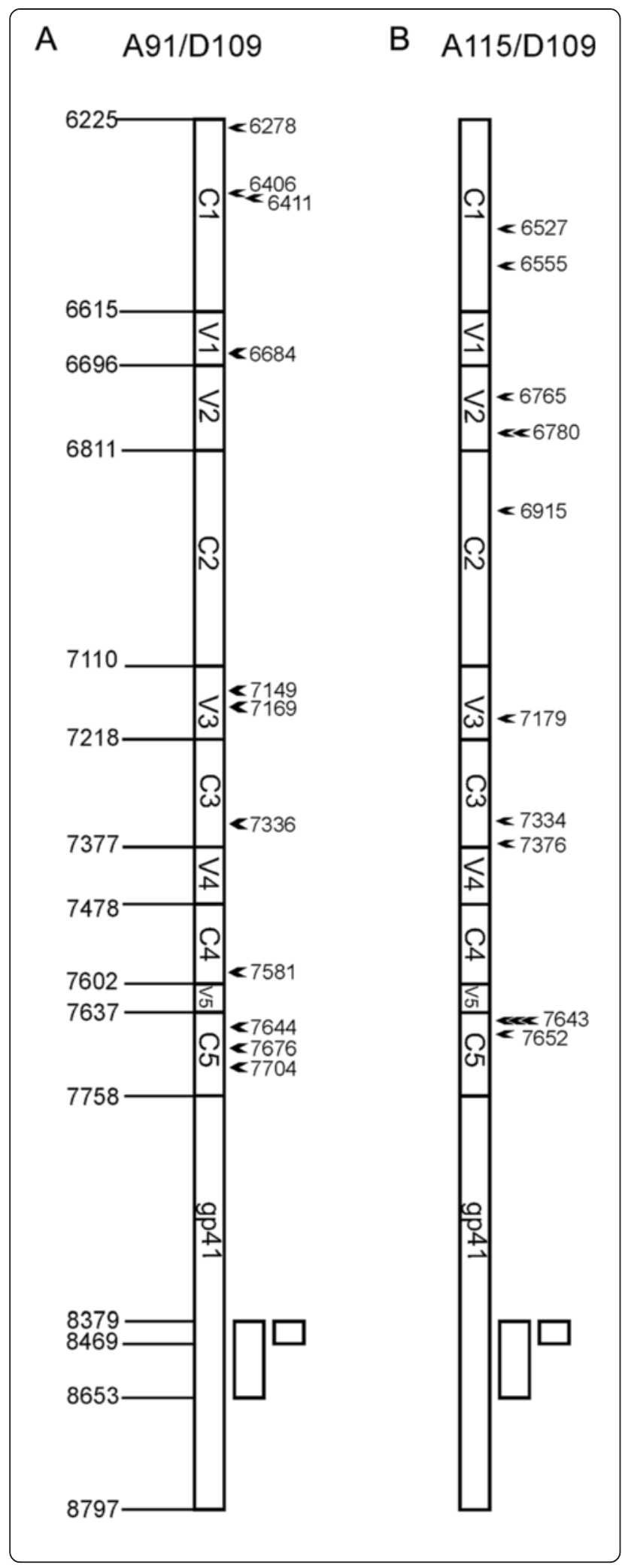

Fig. 1 Distribution of recombination breakpoints in gp120 region. Clonal gp120 recombinant sequences were aligned with parental strains and the recombination breakpoints were determined using similarity plots constructed in SimPlot sequence analysis software. Distribution of breakpoint in gp120 region for A91/D109 and A115/ D109 dual infection sets is indicated in (a) and (b) respectively

(11 A91/D109 and 10 A115/D109) in pREC_nfl_HIV NL43 did not mediate cell-to-cell fusion (Fig. $3 \mathrm{~b}$ and c). A similar outcome was observed with the respective recombinant viruses. Spreading virus infections were observed with the NL4-3 containing the parental A91, A115 and D109 HIV-1 env genes (Fig. 3d) whereas the viruses with the env-gp120 A/D recombinants were replication defective (Fig. 3e and f).

\section{Impact of chimeric Tat/Rev proteins on replication of $A / D$} recombinant viruses

HIV-1 Tat and Rev proteins are translated from two exons. The first exons of tat and rev are located upstream of the env gp120 coding region, while tat 2 and rev2 exons are found in the gp 41 coding region. In the pREC_nfl_HIV constructs containing the env-gp120 A/D recombinants, tat1/rev1 exons are of NL4-3 sequence and spliced to tat2/rev2 exons of D109 sequence resulting in chimeric Rev and Tat proteins. Thus, defective pREC_nfl_HIV constructs with env-gp120 A/D may be the consequence of non-functional, chimeric Tat/Rev accessory proteins rather than the direct consequence of a chimeric A/D Env glycoprotein. To rule out this possibility, we replaced the NL4-3 tat1 and rev1 exons in the backbone with the corresponding sequence from isolate D109 such that both tat/rev exons would be derived from the same subtype D HIV-1 isolate in the env-gp120 A/D recombinants (see Methods) (Fig. 2b). Two of the 21 env-gp120 A/D recombinants, one from the set of A91/D109 (Fig. 4b and e) and one from A115/D109 (Fig. 4c and $\mathrm{f}$ ), were functional upon introduction of the concordant D109 tat1 and rev1 exons. This result suggests that in some instances, chimeric A/D Tat and Rev proteins can restrict or reduce HIV-1 replication of some intersubtype recombinants with breakpoints appearing in the gp120 coding region of env. However, the majority of env-gp120 A/D recombinants remained non-functional indicating that sequence concordance in the first and second exon of tat/rev is not the primary cause of this restriction. Please note that $v p u$ in the cloned HIV-1 viral genome is also chimeric $(\mathrm{B} / \mathrm{A})$. However, the constructs with the primary envs containing the same $v p u$ produced replicative viruses (Fig. 3a and d) suggesting that it has no significant influence in the replication capacity. 


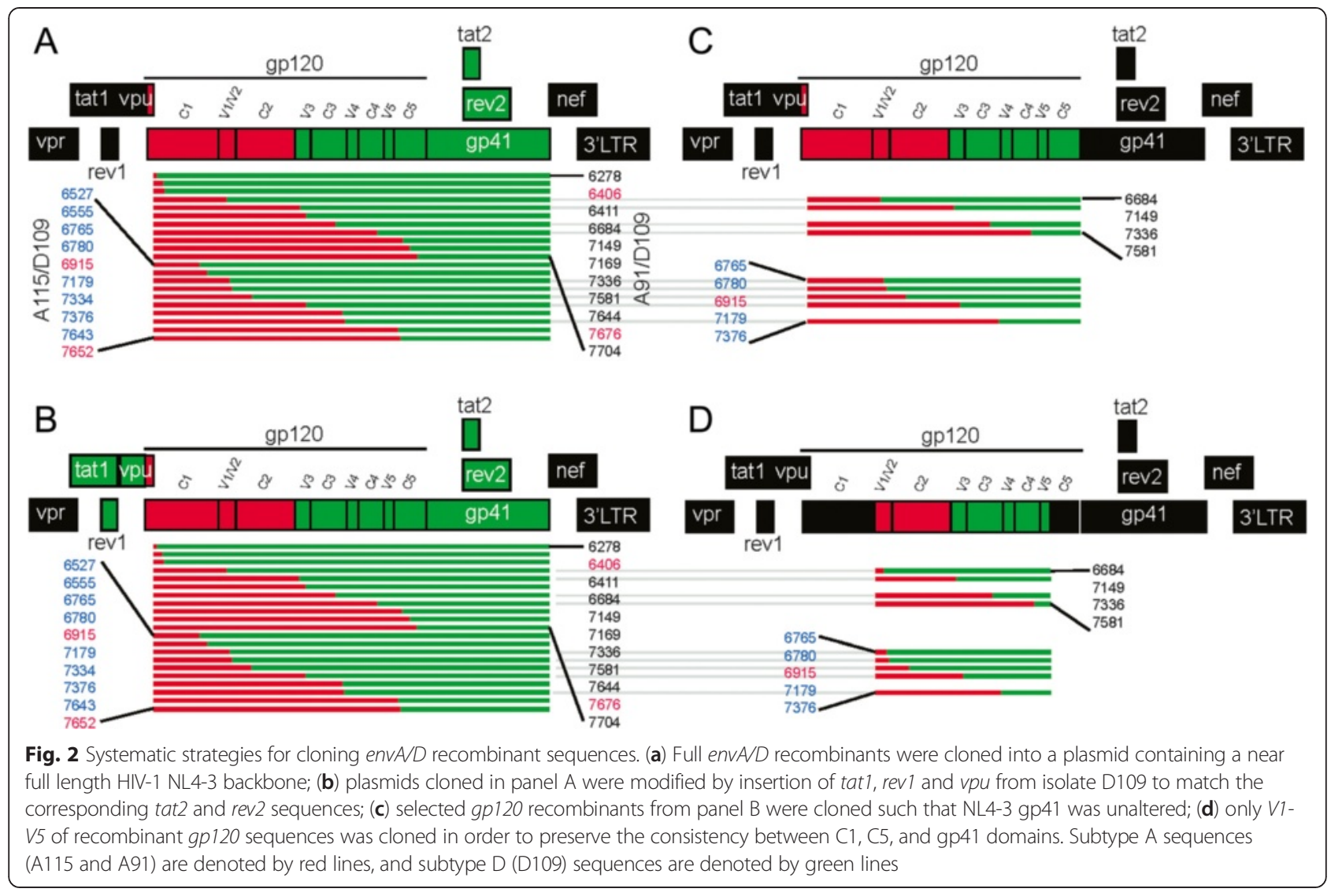

\section{Impact of $A / D$ gp120 coding region on replication of $A / D$} recombinant viruses

The gp120 region of the HIV-1 envelope is responsible for CD4 binding, coreceptor recognition, immunogenicity, immune evasion, interactions with gp41, and coordinating the host cell entry process. Thus, intersubtype recombination in gp120 can result in a significant shift in genetic differences and could result in a non-functional envelope on the viral surface. By cloning just the A/D gp120 region into the NL4-3 backbone, there was no disruption of another HIV-1 coding region and we could retain tat and rev genes from the NL4-3 backbone. Thus, we could examine the impact of intersubtype recombination sites on gp120 function (in association with other interacting proteins, e.g. gp41). On the other hand, based on possible interactions between the $\mathrm{N}$ and C-terminus of gp120 in the crystal structure [36-38], we suspect that the function of A/D recombinants with breakpoints in V1-V5 domains might be maintained if retaining the NL4-3 C1 and C5 domains. Cloning the parental A91, A115 and D109 V1-V5 region into the NL4-3 backbone resulted in functional Env and replication competent virus suggesting again that this subtype B NL4-3 virus could accommodate a diverse chimeric Env glycoprotein.

For these analyses, we cloned either the entire gp120 coding region or only V1-V5 region of 9 A/D gp120 recombinants with breakpoints in gp120 (bk6684, bk7149, bk7336, and bk7581 from A91/D109, and bk6765, bk6780, bk6915, bk7179, and bk7376 from A115/D109), all containing breakpoints within V1-V5 regions into NL4-3 backbone (Fig. 2c and 2d). We excluded the analyses of gp120 A/D recombinants which contained breakpoints either in C1 or C5 region (i.e. bk6278, bk4406, bk6411, bk7169, bk7644, bk7676, bk7704, bk6527, bk6555, bk6915, bk7334, bk7643, and bk7652). The cloning of the entire gp120 coding regions of these 9 A/D gp120 recombinants resulted in none of functional Env glycoprotein by Veritrop or replication defective virus (Fig. 5a and b). Please note that all 9 A/D gp120 coding regions cloned into NL4-3 backbone resulted in production of recombinant Env glycoproteins on the viral surface as demonstrated by EIA using plates coated with anti-gp120 antibody B13 (Fig. 5c), as well as Western blot (Fig. 5d). Interestingly, 4 out of 9 A/D V1-V5 regions cloned into an NL4-3 backbone (in between the C1 and C5 regions of NL4-3) resulted in functional Env glycoproteins and replication competent viruses (Fig. 6a and b). Although bk6684 (breakpoint at V1/V2 region), bk7149 (V3), bk7336 (C3), and bk7376 (C3) could not replicate or produce functional Env when cloned as a whole Env or gp120, the cloning of V1-V5 region of these A/D recombinants successfully rescued their function (Fig. 6a and b). 


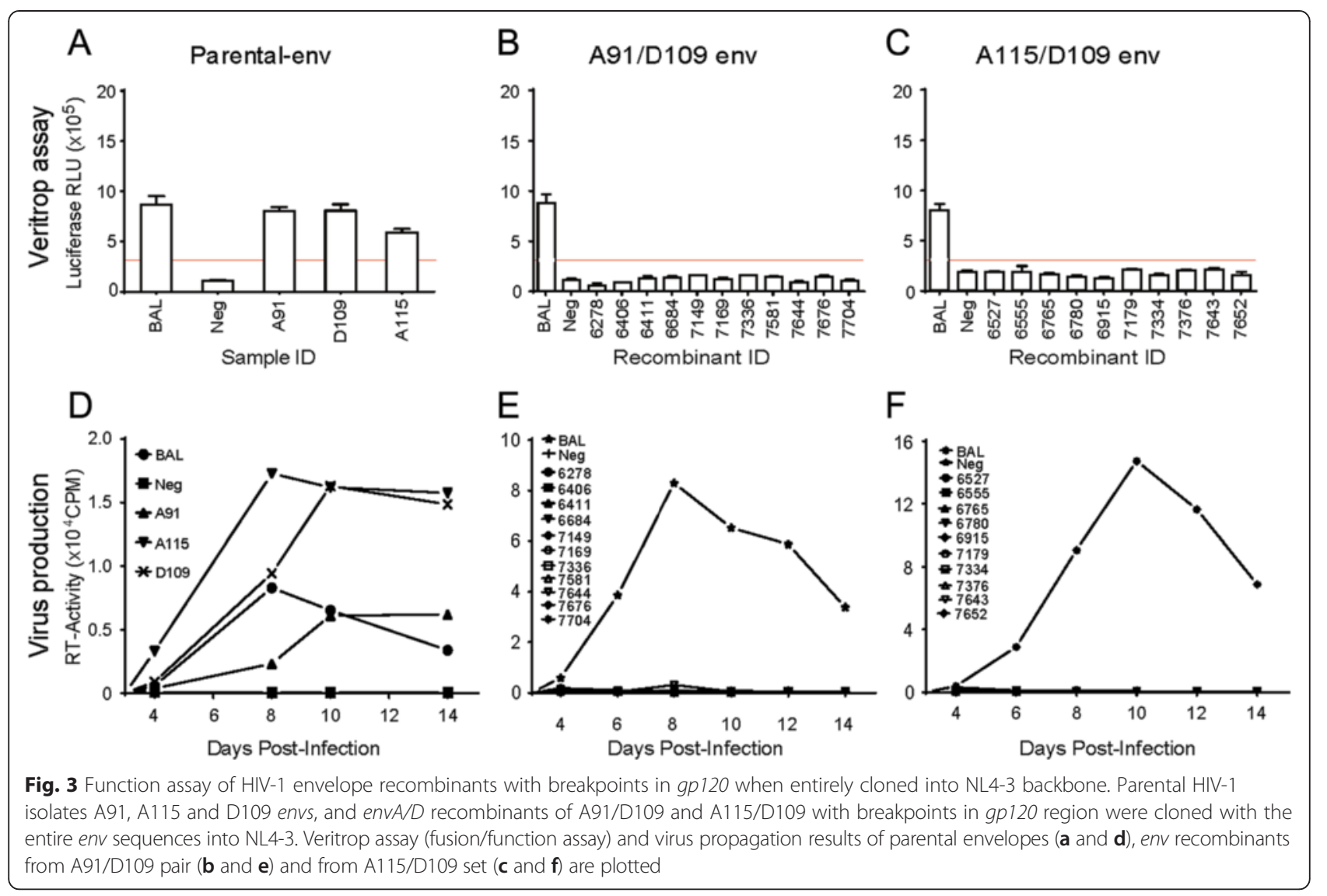

\section{Co-evolution and structural interactions between gp120 C1, C5, and ecto-gp41 domains}

Our studies on intersubtype recombination in env as well as sequence analyses of patient-derived URFs and CRFs revealed that breakpoints are rarely observed in the V1-V5 gp120 coding region. There are only 4 listed A/D CRFs (and 1 A1DG), and 2 of these are A/D in Env. CRF35_A1D Env is A1, with a small region in the C4/C5 region being subtype D, while CRF50_A1D has breakpoints between C1 and C3 (http://www.hiv.lanl.gov). Again, when Env function is required, recombination events in env tend to cluster in the leader-C1 region of gp120 or in the transmembrane domain of gp41 [30, 31]. In the absence of functional selection, intersubtype recombination is quite prevalent in the other conserved regions $\mathrm{C} 2, \mathrm{C} 3$, and $\mathrm{C} 4$ and still evident in the hypervariable sequences. Previous studies indicate that cross-overs/breakpoints can occur anywhere on the template during reverse transcription but are more frequent at regions of higher sequence conservation [30, 31].

The absence of functional intersubtype recombinants with breakpoints between the $\mathrm{C} 1$ and $\mathrm{C} 5$ domains of HIV-1 gp120 may be associated with co-evolving interactions between the linear ends of the gp120 and with the ecto domain of gp41. Galli A, et al. studied the recombination in pol region between two subtype $B$ sequences (B/B) and between one subtype $\mathrm{B}$ and one subtype $\mathrm{F}$ sequence $(\mathrm{B} / \mathrm{F})$, and found the evidence for the evolved coadapted sites in variants from different subtypes [39]. Numerous studies have mapped possible interaction between the gp120 C1 and C5 domains with the ecto domain of gp41, which is now supported by a recent $4.7 \AA$ crystal structure of a soluble, cleaved BG505 SOSIP.664 gp140 trimer in complex with a potent broadly neutralizing antibody (bnAb), PGT122, and recent $5.8 \AA$ A cryo-electron microscopy re-construction of the Env trimer in complex with a CD4 binding site bnAb, PGV04 [36-38, 40-44]. In Fig. 7, we have utilized the crystal structure coordinates of the Env trimer (PDB - 4NCO) to highlight the interaction between the gp120 C1, C5, and ecto-gp41 domains (residues 1-126). For this model, the bnAb PGT122 is hidden, viral membrane would be on top, and CD4/CCR5 would engage at the bottom of each view (Fig. 7a). The Env trimer "core" containing the close association between $\mathrm{C} 1$, C5, and ecto-gp41 domains of each gp120 subunit is shown by turning the structure $90^{\circ}$ clockwise (Fig. $7 \mathrm{~b}$ ) and by being stripped of all other gp120 residues (Fig. 7c). These trimer domains are less exposed and more encased by the remaining gp120 regions (Fig. 7d). 


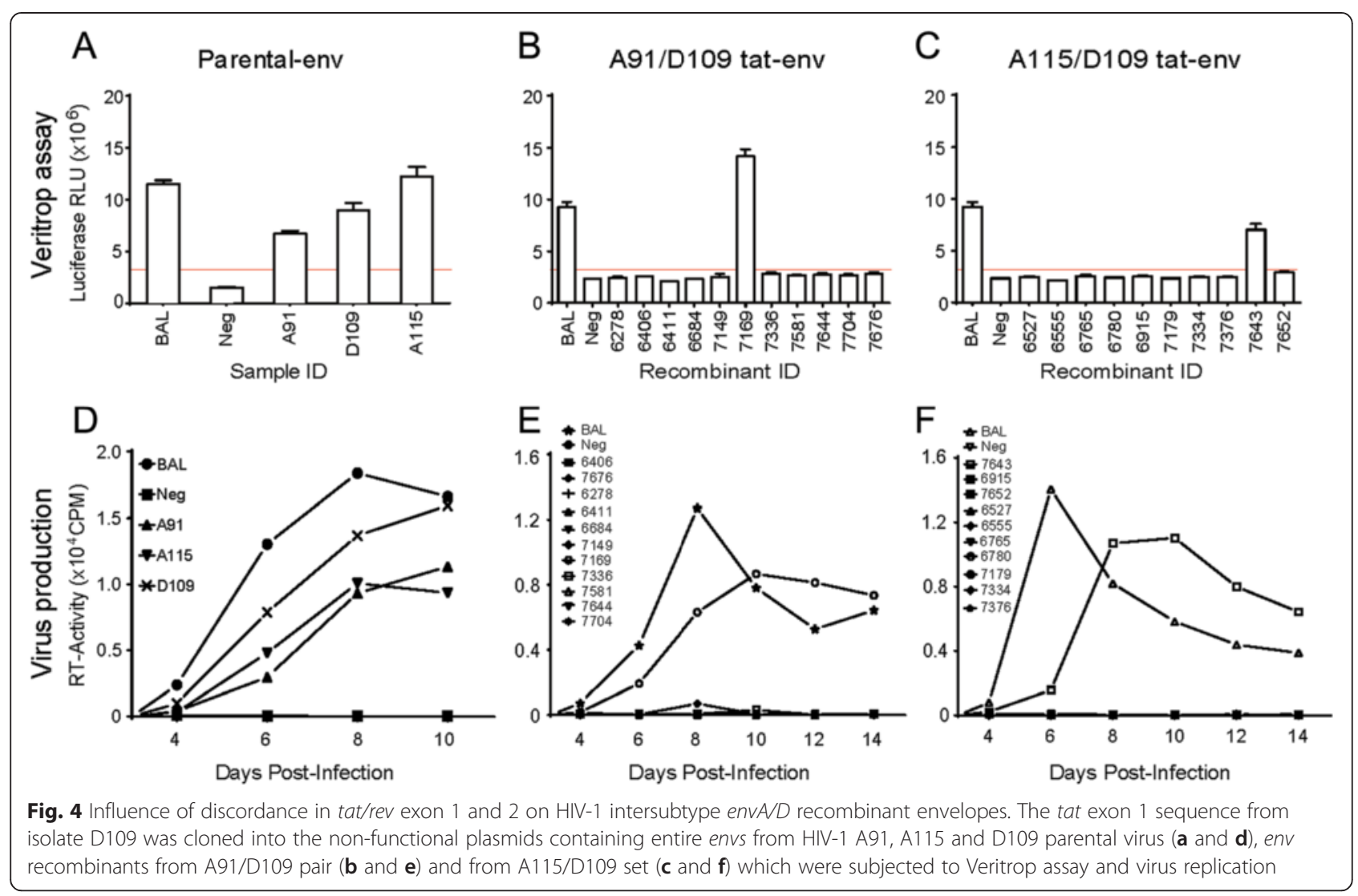

Analyses of the Env trimers suggest a close interaction between $\mathrm{C} 1, \mathrm{C} 5$, and ecto-gp41 domains despite the $\sim 340$ amino acids separating this $\mathrm{N}$ and $\mathrm{C}$ terminus in the linear sequence. Thus, lack of "functional" intersubtype recombinations with breakpoints between the $\mathrm{C} 1$ and C5 domains may be related to the inability of the subtype A C1 domain to interact with the subtype D C5 and ecto-gp41 domains. If this is the case, we should observe co-evolution between the $\mathrm{C} 1$ and C5/ecto-gp41 domains within a subtype. As described in the Methods and Fig. 8, we constructed separate maximum likelihood trees on the coding regions of $\mathrm{C} 1, \mathrm{C} 2, \mathrm{C} 3, \mathrm{C} 4, \mathrm{C} 5 /$ ectogp41 domains using 144 and 97 unique subtype A1 and D Env sequences (100 bootstrap replicates for each), and using the rtREV [45] model for phylogenetic inference of amino acid substitutions. We then compared the tree topology of each matched terminal taxa in the tree for all pairs of conserved Env regions (e.g. $\mathrm{C} 1+\mathrm{C} 2, \mathrm{C} 1+\mathrm{C} 3$, $\mathrm{C} 1+\mathrm{C} 4, \mathrm{C} 1+\mathrm{C} 5 /$ ecto-gp41, etc.) using Mirrortree web server [46] which was developed to predict functional relationships between protein families. In other words, relative genetic distances between all 144 branches of the subtype $\mathrm{A} 1$ tree of $\mathrm{C} 1$ domain are compared to that of the subtype A1 tree of C2 domain. Heatmaps demonstrating the correlation between each paired domain in Env were produced with Plot (https://plot.ly). To simplify, the strongest co-evolution between two conserved Env domains would have the best overlay between tree topology of those conserved regions and show the strongest correlation. With both subtype A1 and $\mathrm{D}$, the $\mathrm{C} 1$ domain shows the strongest co-evolution with the C5/ecto-gp41 domain $(r=0.44$ and 0.47 , respectively) (Fig. 9a and c) comparing with other regions, e.g. C3 domain (Fig. 9b, and d). Interestingly, linear order of the conserved regions does not exhibit consistent evidence of co-evolution along the length of the gene, indicating that the proximity of the regions to each other was not the driving factor of covariance (i.e. coevolution). For example, C1 is separated from C2 by $\sim 66$ amino acids (V1/V2) and shows a moderate pattern of covariance $(r=0.26-0.39)$, while $\mathrm{C} 3$ and $\mathrm{C} 4$, who are separated by a much shorter distance of $\sim 11$ amino acids, show a weak pattern of covariance $(r=0.07-0.22)$.

To further predict protein co-evolution, we used the methods developed in Pazos and Valencia [47], which are similar to the methods implemented in mirrortree with the exception that phylogeny of the viruses is not taken into consideration since correlation is based solely on the underlying distance matrices. The Jones-TaylorThornton (JTT) matrix-based model [48] uses a uniform substitution rate among sites to calculate the number of amino acid substitutions per site between each sequence 


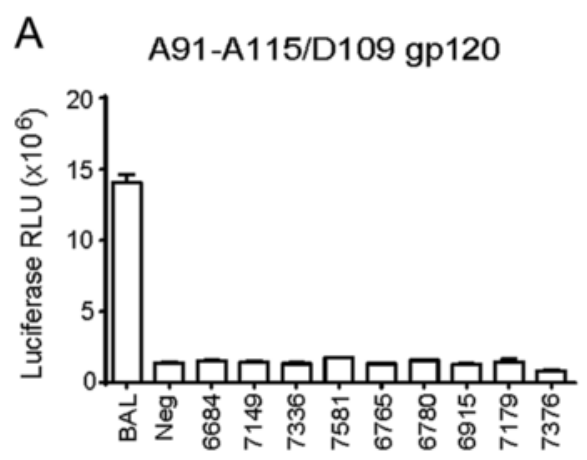

C

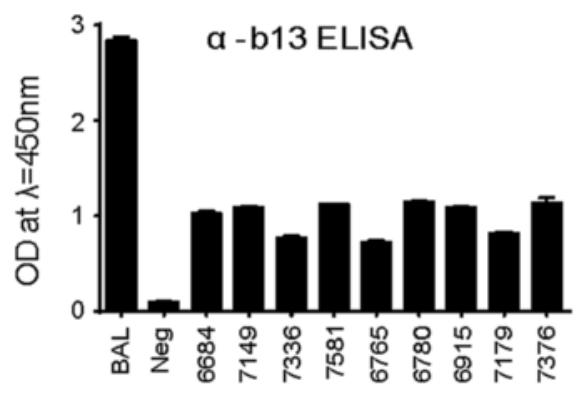

Recombinant ID
B

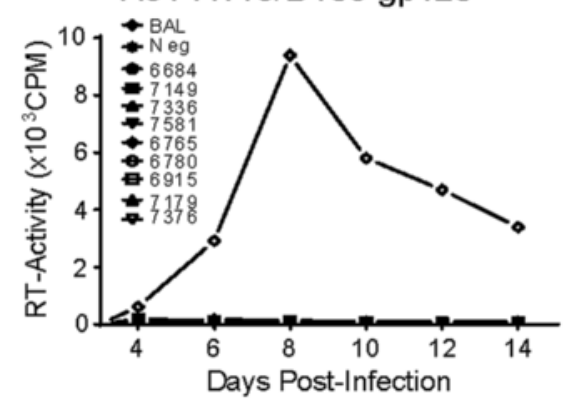

D

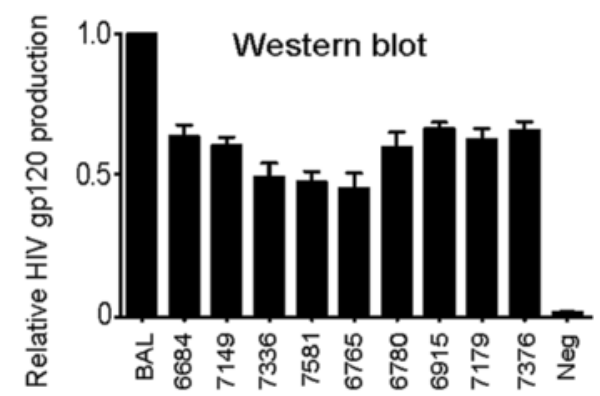

Recombinant ID

Fig. 5 Cloning of only gp120 sequences of intersubtype envAVD recombinants into NL4-3 backbone and the resultant envelope function assay. Intersubtype A/D recombinant gp120 genes were cloned, leaving gp41 of NL4-3 intact. (a) The plasmids containing recombinant gp 120 sequences were tested by Veritrop assay for functionality and (b) for replication-competent virus production. Pseudoviruses produced from the plasmids were tested by ELISA (c) and Western blot (d) for the production of envelope expression

in the alignment. The pairwise distances were calculated for paired conserved region $(\mathrm{C} 1, \mathrm{C} 2, \mathrm{C} 3, \mathrm{C} 4$, and $\mathrm{C} 5$ gp41) of every virus in the multiple sequences, generating 10,296 and 4656 data points for subtype A1 and D viruses. Pearson's product-moment correlation coefficient was calculated to measure the relationship between the distances paired conserved regions for these viruses. As in the analysis of the maximum likelihood tree topology described above, the strongest correlations existed between $\mathrm{C} 1$ and $\mathrm{C} 5$-gp41 (Fig. 9e and f).

\section{Discussion}

We and others have mapped intersubtype recombination in the env gene using various HIV-based systems. The frequency and site of intersubtype recombinations is governed by sequence conservation, RNA secondary

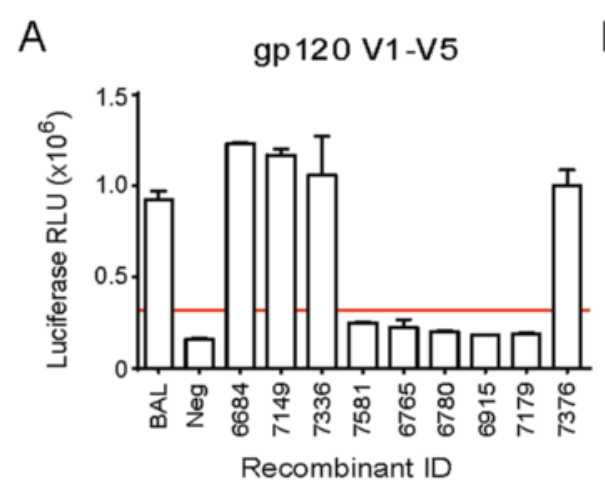

B

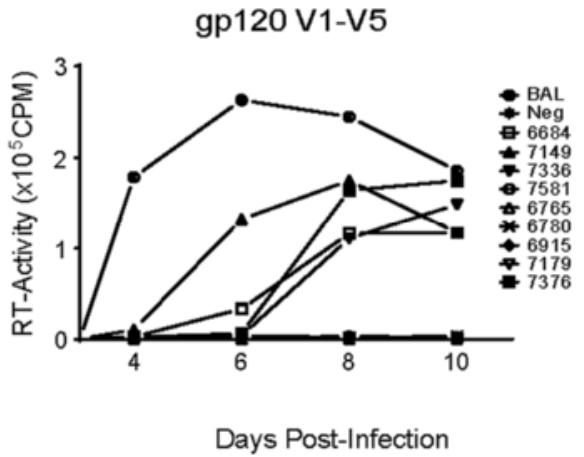

Fig. 6 Cloning of only V1-V5 domains of envAVD recombinants into NL4-3 backbone and the resultant envelope function assay. The V1-V5 domains of 9 enVAVD recombinants were cloned into NL4-3 backbone, and were tested for their functionality by Veritrop assay (a) and for their ability to produce replication-competent viruses $(\mathbf{b})$ 


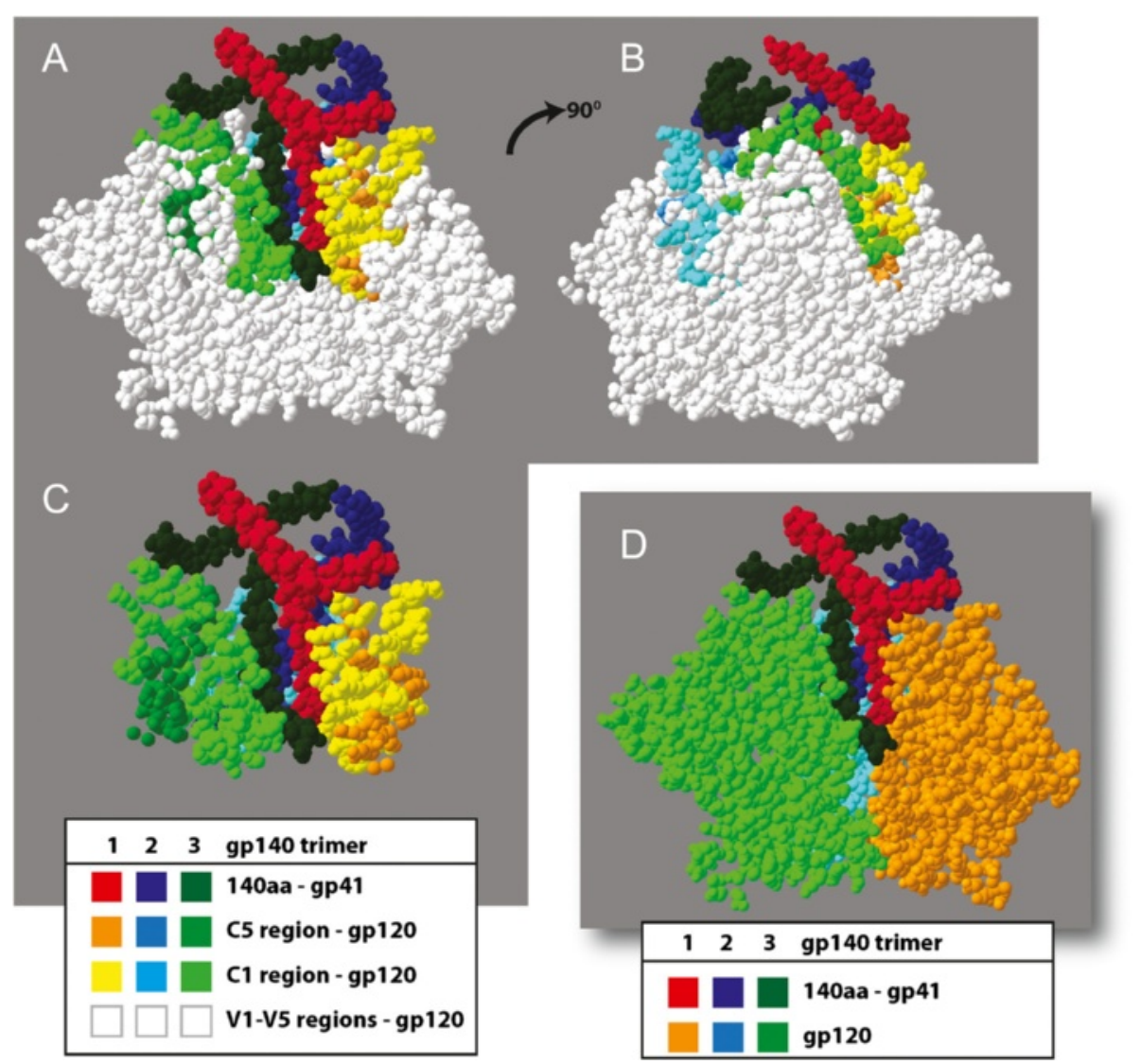

Fig. 7 Model of the C1/C5/ecto-gp41 core in the crystal structure of the HIV-1 gp140 trimer. This figure is derived from the recent $4.7 \AA$ crystal structure of a soluble, cleaved BG505 SOSIP.664 gp140 trimer in complex with a potent bnAb, PGT122 [37] (PDB - 4NCO). The interaction between the gp120 C1, C5, and ecto-gp41 domains is highlighted. For this model, the bnAb PGT122 is hidden, viral membrane would be on top, and CD4/CCR5 would engage at the bottom of each view. The Env trimer "core" of the C1, C5, and ecto-gp41 of each gp140 subunit is shown in panel (a), turned $90^{\circ}$ in (b), and stripped of all other gp120 residues in panel (c). Finally, each gp140 subunit of the trimer is shown in panel (d)

structures, and selective pressure for subsequent virus replication [1, 30, 33, 49, 50]. However, few studies [1, $33,51]$ have characterized the function of these intersubtype recombinant genomes generated in vitro. Our laboratory has developed several systems to understand the mechanisms involved in HIV-1 recombination. Early studies explored the generation of intersubtype HIV-1 recombinants in the env gene by dually infected susceptible cell cultures with HIV-1 subtypes that co-circulated in regional epidemics [31, 32]. Dual infection generally produces intersubtype recombinant viruses at low frequency that are defective or that cannot compete with the parental viruses. To enrich for "functional" recombinants, we utilized HIV isolate-specific siRNAs that prevented outgrowth of parental viruses while permitting replication of intersubtype recombinants with breakpoints that "recombined out" the siRNA target sequences [2]. With both studies, the preliminary evidence still suggested that intersubtype breakpoints in gp120 led to non-functional envelopes and defective viruses.
However, we did not employ a systematic approach to investigate the impact of intersubtype recombination in the env gene on function and support of HIV-1 replication. The mechanism of HIV-1 intersubtype recombination during reverse transcription does not, of course, account for the functionality of the resulting genomes, which is largely controlled by the minus strand DNA invasion onto another HIV-1 RNA genome during pausing of reverse transcriptase at homopolymeric tracts or RNA secondary structures. Again, these strand transfer events occur at increased frequency in regions of high sequence conservation $[1,30]$, which could lead to selection of more functional intersubtype. Based on this process, intersubtype recombination can often result in split codons (i.e. non-synonymous amino acid substitutions), insertions, deletions, and substitutions that can change the open reading frames and/or introduce premature stop codons.

In this study, we have screened out all of the intersubtype recombination events in env that obviously impact 


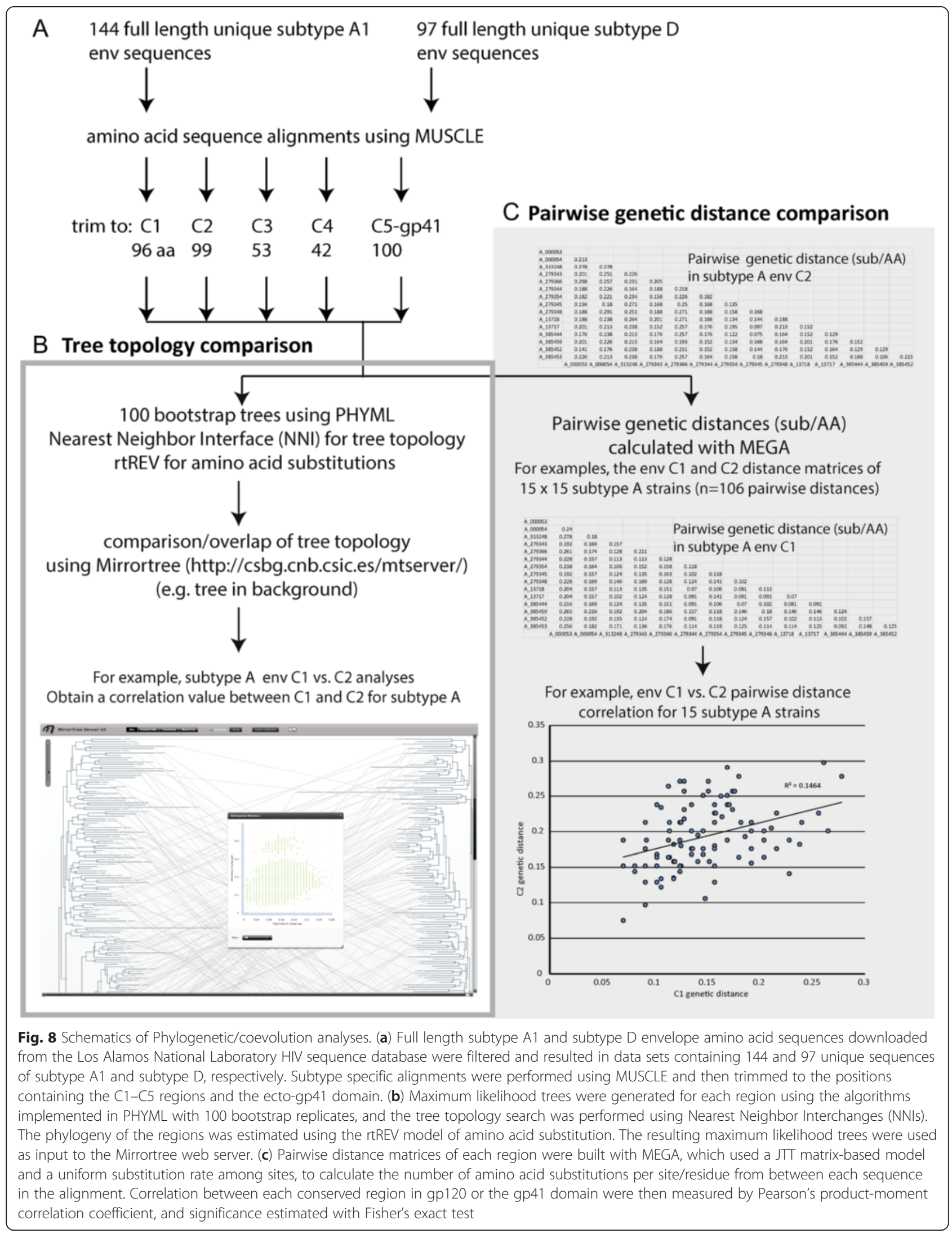




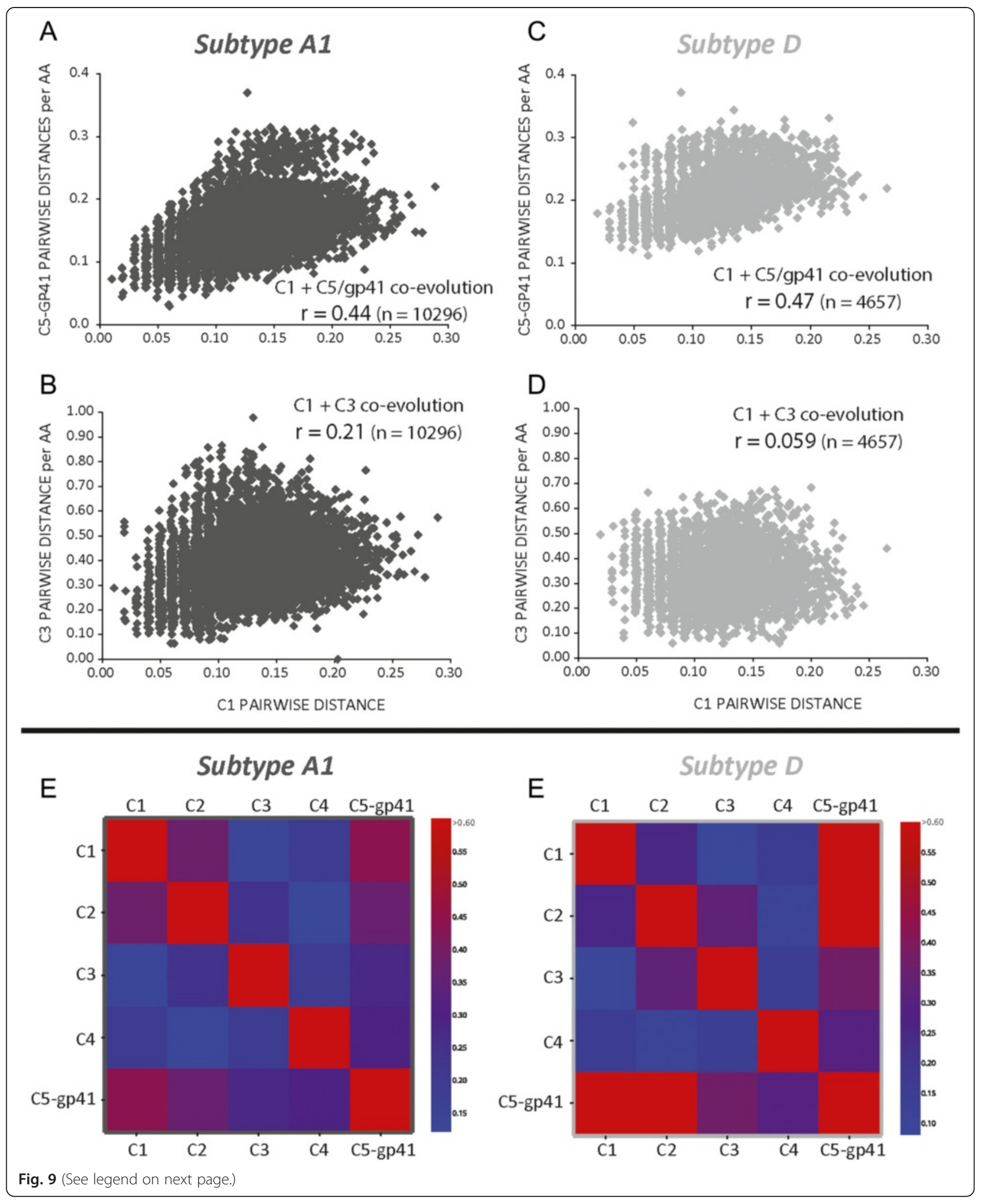


(See figure on previous page.)

Fig. 9 Co-evolution of the C1/C5/ecto-gp41 domains in HIV-1 subtype A and D isolates. Scatter plots demonstrating the correlation between the pairwise distance matrices of viral isolates were built from multiple sequence alignments of unique subtype $A 1$ and subtype $D$ env sequences $(\mathrm{n}=10,296$, (a) and (b); $n=4657$ (c) and (d), respectively). Pairwise comparisons were performed with all pairs of conserved C1, C2, C3, C4, and C5-gp41 regions but only the comparison of $\mathrm{C} 1+\mathrm{C5} / \mathrm{gp} 41$ ( $\mathbf{a}$ and $\mathbf{c}$ ) and of $\mathrm{C} 1+\mathrm{C} 3$ (b and $\mathbf{d}$ ) are presented in this figure. Pearson product moment correlations are shown for each panel but due to high number of sample sequences, $p$ values were highly significant $(<0.0001)$ for all paired analyses suggesting some level of co-evolution throughout the protein as expected. The heatmaps in (e) and (f) reflect the tree correlation coefficients derived from a similarity comparison of the maximum likelihood derived trees of the multiple sequence alignments of the subtype specific conserved regions. The correlation coefficients between C1 and C5-gp41 demonstrate the strongest similarity of tree topology among the regions compared

protein expression. Instead, we focused on the intersubtype recombination events with intact open reading frames that Env expression may or may not generate a functional chimeric protein. The HIV-1 env gene contains tat 2 and rev2 exons that must be precisely spliced and joined to tat 1 and rev1 exons to produce functional Tat and Rev proteins [26, 27]. There is a significant intersubtype sequence variability in both tat and rev sequences and there is evidence of co-evolution within the first and second exons of these accessory genes as well as within the TAR and RRE RNA target sequences [28, 29]. A recombination breakpoint in the gp120 coding sequence would result in an intersubtype discordance in tat 1 and tat 2 or rev1 and rev2, which impact Tat and Rev expression as well as their function. Intersubtype A/ $\mathrm{D}$ recombination in gp120 did not result in a frameshift or premature stop codon due to combination of discordant subtypes in the first and second exons. However, there was the possibility that discordant subtype sequences for the first and second exons of tat/rev impaired their function. In the present study, we found that two non-functional A/D env recombinants clones (out of 21) in an NL4-3 backbone were "rescued" for host cell entry and virus replication when "matching" tat/rev exons 1 and 2 were introduced. Interestingly, the other $19 \mathrm{~A} / \mathrm{D}$ env recombinants clones in an NL4-3 backbone with the same functional Tat/Rev proteins remain non-functional, even though the Env glycoproteins were observed in all of these A/D recombinant viruses, suggesting that the discordance of tat $1 /$ rev1 and tat $2 /$ rev 2 might not play the main role in restricting the function of resultant HIV-1 A/D intersubtype Env recombinants.

In the recombinant virus with surface expression of the A/D envelope glycoprotein, the lack of replication and host cell entry was most likely attributable to impaired function of this chimeric protein. Previous studies have described the possible non-covalent interactions between gp120 and gp41. The topology of the gp120 core structures implies that gp120 C1 and C5 are in close proximity [52-54], and immunological studies have also suggested interactions between the $\mathrm{C} 1, \mathrm{C} 5$, and the gp41 disulfide loop [23, 55]. The gp41 components are membrane-proximal and interspersed with the gp120 C1 and C5 elements. These two HR1 helices pack against hydrophobic gp120 residues in $\mathrm{C} 1$ and $\mathrm{C} 5$. The close proximity of $\mathrm{C} 1$ and $\mathrm{C} 5$ elements most likely participates in tertiary structure interactions with residues connecting HR1 to HR2 $[40,56]$. The thermodynamic stability of this gp120-g41 interface is essential for the integrity of HIV-1 envelope trimer structure and function [40, 57]. The non-covalent nature of gp120-gp41 interaction presents significant challenges, for this contact must be of sufficient flexibility to maintain subunit interactions following gp120 binding to CD4 and subsequent gp120 conformational changes that promote its interaction with the CCR5 or CXCR4 co-receptor. The authenticity of this gp120-gp41 interaction for function is documented in experiments describing disruption due to minor temperature change, antibody or soluble CD4 binding, and inhibitor binding [57-59]. Based on these findings, we investigated if the expressed but nonfunctional Env was due to subtype discordance between the $\mathrm{C} 1$ and C5/ecto-gp41 domains introduced by intersubtype recombination in the gp120 coding region. First, cloning of the A/D gp120 coding region did not restore Env function or virus replication for any of the 9 selected clones in the NL4-3 backbone. However, the cloning of just V1-V5 regions resulted in a gain of function/ replication for 4 of these $9 e n v A / D$ recombinant viruses. In this situation, the $\mathrm{C} 1, \mathrm{C} 5 / \mathrm{gp} 41$ regions were retained from NL4-3 but V1-V5 gp120 sequences contained an upstream subtype A coding region fused to a subtype D coding region. Despite the extreme chimeric nature of the Env glycoprotein (e.g. subtype B-C1, A-V1 to V3, DC4 to V5, B-C5 and B-gp41 for clone bk7169), many of these viruses with the A/D gp120 V1-V5 regions were functional. In contrast, a virus encoding the recombinant breakpoint as an Env cassette (e.g. subtype A-C1 to V3 and subtype D-C4 to gp41 for clone bk7169) could not mediate host cell entry and could not replicate despite having Env expression on the viral particle surface. Interestingly, Simon-Loriere et al found that defective intersubtype recombinants were most frequently observed in the 
$\mathrm{C} 2$ and $\mathrm{C} 3$ domains of $\mathrm{C} / \mathrm{B}, \mathrm{A} / \mathrm{C}$, and $\mathrm{G} / \mathrm{B}$ env recombinants and less so in $\mathrm{A} / \mathrm{G}$ and $\mathrm{A} / \mathrm{B}$ env recombinants. Those nonfunctional intersubtype recombinants with $\mathrm{C} 2$ and $\mathrm{C} 3$ breakpoints may also be related to the coevolution and interaction of the $\mathrm{C} 1, \mathrm{C} 5$ and gp41 domains [60].

The importance of this $\mathrm{C} 1 / \mathrm{C} 5 /$ ecto-gp41 interaction for Env function is best supported by the recent $3 \AA \mathrm{X}$ ray crystal structure of gp140 trimer in complex with a potent bnAb, PGT122 and the $5.8 \AA$ cryo-electron microscopy re-construction of the Env trimer in complex with a CD4 binding site bnAb, PGV04 [36-38, 40-44]. In this study, we have utilized the available crystal structure coordinates to visualize this $\mathrm{C} 1 / \mathrm{C} 5 /$ ecto-gp41 interaction within the gp140 trimer. As described in Fig. 7, the $\mathrm{C} 1 / \mathrm{C} 5 /$ ecto-gp41 domains wrap together to form an inner core within the gp140 trimer. Thus, it is not surprising that major genetic shifts within this structure (e.g. intersubtype recombination events) may prevent proper Env function. This highly conserved structural motif may also be one of the reasons for the paucity of intersubtype recombination breakpoints in the gp120 coding region within the HIV-1 population but significant hot spots for recombination prior to the $\mathrm{C} 1$ region and following the ecto-gp41 domain of Env (http:// www.hiv.lanl.gov/).

The close interaction between the C1/C5/ecto-gp41 domains within Env glycoprotein trimers suggests a possible co-evolution (co-variance) within these domains during HIV-1 divergence in the human epidemic. We specifically analyzed env sequences from a representative set of 144 and 97 unique HIV-1 subtype A1 and D isolates (http://www.hiv.lanl.gov) using two maximum likelihood/matrix models to test for co-evolution among gp120-C1,-C5, and ecto-gp41 domains. Based on these models, $\mathrm{C} 1$ and C5/ecto-gp41 domains had higher covariance/co-evolution than any other paired gp120/gp41 regions (e.g. $\mathrm{C} 1+\mathrm{C} 2, \mathrm{C} 1+\mathrm{C} 3, \mathrm{C} 1+\mathrm{C} 4$, etc.). Previous mutational analyses revealed evidences for $\mathrm{C} 1, \mathrm{C} 5$ and ecto-gp41 interactions. For example, V44A and F53A mutants can disrupt gp120/gp41 interactions [44] whereas gp120 mutations V36L and Y40D resulted in dissociation of gp120 from gp41 [61] despite interactions with cellular CD4 receptor [61]. C1 and C5 domains of gp120 form the two alpha helices that constitute the hydrophobic inner core that directly interacts with the immunodominant loop in the gp41 ectodomain [40, 42, 44]. Mutational studies revealed interactions between I491 of C5 and W596/S618 of the gp41 immunodominant loop [42, 62] and an additional A501 of C5 interacting with T605 of this gp41 loop [40]. Biochemical mapping of these C1/C5/ecto-gp41 interactions have now been confirmed with the recent gp140 trimer structure, which now suggests that many of these interactions are inter- as opposed to intramolecular interactions within the trimer $\mathrm{C} 1 / \mathrm{C} 5 /$ ecto-gp41 core. Based on the present study, we cannot assume that breakpoints within the gp120 region would result in non-functional gp120 recombinants between other subtypes. However, with selection pressure for replication competency, we have rarely observed breakpoints within the gp120 of other intersubtype recombinants (http://www.hiv.lanl.gov/). Through analyzing 77 functional various intersubtype recombinants with single breakpoints in the env coding region, we have found that only $5 \%$ have recombination sites in gp120. We are currently exploring the possible linked amino acids in the $\mathrm{C} 1, \mathrm{C} 5$ and ectogp41 domains that may interact in the gp140 trimer. As opposed to just structure-based mutagenesis studies, we can also identify key amino acid residues and structures in the trimer $\mathrm{C} 1, \mathrm{C} 5$ and ecto-gp41 core using a combination of co-evolution studies along with analyses of functional intersubtype recombinants in env.

\section{Conclusions}

Studies on the gp120/gp41 structure of HIV-1 intersubtype Env recombinants could be the key to understanding the labile $\mathrm{C} 1 / \mathrm{C} 5 /$ ecto-gp 41 interface and could help in design of drugs to disrupt this interaction. Our study on HIV-1 env gene evolution in the epidemic reveals a strong link and co-evolution between the C1, C5 and ecto-gp41 domains which is less apparent between other subdomains of gp120 and gp 41 . These findings suggest a strong selective pressure for co-evolution in the $\mathrm{C} 1, \mathrm{C} 5$ and ecto-gp41 domains to maintain Env function and virus replication. In this study we have identified two mechanisms responsible for the genetic bottlenecks that select for specific replication-competent intersubtype Env recombinants. Of course, this was following the exclusion of the obvious that prevented Env expression, i.e. premature stop codons, insertions/deletions, and frameshifts. First, recombination within the gp120 coding region can generate non-functional or defective Rev and Tat proteins due to subtype/isolate discordance between the first and second exons of these accessory genes. Second, recombination within the gp120 coding region can introduce subtype/isolate discordance that disrupts the highly conserved C1/C5/ecto-gp41 interactions involved in the gp120/gp41 interface and critical for host cell entry.

\section{Methods}

\section{HIV-1 primary isolates}

A91 and A115 are HIV-1 subtype A strains, and D109 is HIV-1 subtype D strain, and they were all CCR5-tropic viruses. These viruses were collected from treatment-naive HIV-1-infected pediatric patients under IRB approval and patients consent through Joint Clinical Research Center 
(JCRC) in Kampala, Uganda in 1996. Neither patients nor their mothers had received any ARV treatment. The viruses were isolated and propagated by co-culturing Peripheral blood mononuclear cells (PBMCs) from the patients and from HIV seronegative blood donors as previously described [63]. PBMCs were obtained by FicollHypaque density gradient centrifugation of heparintreated venous blood. Prior to HIV-1 infection, cells were stimulated with $2 \mu \mathrm{g}$ of phytohemagglutinin (PHA) (Gibco BRL) per ml for 3 to 4 days and maintained in RPMI 1640- $2 \mathrm{mM}$ L-glutamine medium (Cellgro) supplemented with $10 \%$ fetal bovine serum (FBS, Cellgro), $1 \mathrm{ng}$ of interleukin-2 (IL-2) (Gibco BRL) per $\mathrm{ml}, 100 \mathrm{U}$ of penicillin (Cellgro) per $\mathrm{ml}$, and $100 \mu \mathrm{g}$ of streptomycin (Cellgro) per ml. TCID50 assays (tissue culture dose for $50 \%$ infectivity) were performed to determine virus titer. Titers were expressed as infectious units per milliliter [64]. The sequence analysis of env genes of these three HIV-1 isolates were typical subtype A or D viruses.

\section{Cell lines}

U87.CD4.CCR5 cell line was obtained from the AIDS Research and Reference Reagent Program and grown in Dulbecco's modified Eagle's medium (DMEM, Invitrogen) supplemented with $15 \%$ FBS, penicillin and streptomycin, puromycin $(1 \mu \mathrm{g} / \mathrm{ml})$ and G418 sulfate $(1 \mathrm{mg} / \mathrm{ml})$. HEK293 T cells were obtained from the American Type Culture Collection and grown in DMEM supplemented with $10 \% \mathrm{FBS}$ and penicillin and streptomycin. All cells were grown at $37^{\circ} \mathrm{C}$ in $5 \% \mathrm{CO} 2$.

\section{Intersubtype recombination by dual infection}

Production of intersubtype recombinant HIV-1 through in-vitro dual infection has previously been described [32]. One hundred and twenty thousand U87.CD4.CCR5 cells in a 12 well plate were inoculated with 0.1 multiplicity of infection (MOI) of each of primary HIV-1 isolate pair A91 and D109, or A115 and D109. Monoinfections were also set up as controls alongside the dual infections. The viruses were grown for 7 days before culture supernatant was harvested. HIV-1 viral RNA was extracted from the collected supernatant using the QIAamp Viral RNA mini kit (Qiagen), and was immediately used for Reverse transcription PCR (RT-PCR, see below).

\section{Reverse transcription PCR, PCR, and TOPO cloning}

Viral RNA was extracted from $140 \mu \mathrm{L}$ of culture supernatant using the QIAmpViral RNA mini kit (Qiagen), and was converted into cDNA by using Moloney murine leukemia virus RT (M-MLV, Invitrogen) and primer ENV-N (antisense, HXB2 numbering nt9146 to nt9172, 5' -CTGCCAATCAGGGAAGTAGCCTTGTGT-3').

Briefly, $5 \mu$ l of extracted viral RNA was mixed with 40 pmol of primer ENV-N, and cycled for $88{ }^{\circ} \mathrm{C}$ for $2 \mathrm{~min}$, $70{ }^{\circ} \mathrm{C}$ for $10 \mathrm{~min}, 55{ }^{\circ} \mathrm{C}$ for $10 \mathrm{~min}, 3{ }^{\circ} \mathrm{C}$ for $10 \mathrm{~min}$, and $4{ }^{\circ} \mathrm{C}$ hold. Next, $4 \mu \mathrm{l} 5 \times$ first strand buffer (Invitrogen), $2 \mu \mathrm{l} 0.1 \mathrm{M} \mathrm{dTT}$ (Invitrogen), and $1 \mu \mathrm{l} 10 \mathrm{mM}$ dNTPs were added to each reaction and cycled at $25{ }^{\circ} \mathrm{C}$ for $10 \mathrm{~min}, 42{ }^{\circ} \mathrm{C}$ for $2 \mathrm{~min}$, and a $4{ }^{\circ} \mathrm{C}$ hold. Finally, MMLV RT was added to the reaction and cycled at $42{ }^{\circ} \mathrm{C}$ for $1 \mathrm{~h}, 70{ }^{\circ} \mathrm{C}$ for $15 \mathrm{~min}$, and a $4{ }^{\circ} \mathrm{C}$ hold.

To retrieve full length recombinant envs from the culture, nested PCR was performed with external primers ENV A (sense, nt5954 to nt5982, 5' -GGCTTAGGCATC TCCTATGGCAGGAAGAA-3') and ENV M (antisense, nt9068 to nt9098, 5' - TAGCCCTTCCAGTCCCCCCTT TTCTTTTA-3') followed by internal primers Vpu-A-1 (sense, nt6135 to nt6155, 5'-TAGTAGGTATAGAATA TAAGA-3') and gp41-D-2 (antisense, nt8747 to nt8769, 5'-GCCTAATTCTTCTAGGTATGTTG-3'). PCR amplifications were performed with the following conditions: $94{ }^{\circ} \mathrm{C} 4 \mathrm{~min},\left[94{ }^{\circ} \mathrm{C} 30 \mathrm{~s}, 55^{\circ} \mathrm{C} 30 \mathrm{~s}, 72{ }^{\circ} \mathrm{C} 3 \mathrm{~min}\right] \times 35 \mathrm{cy}-$ cles, and $4{ }^{\circ} \mathrm{C}$ hold. Please note, in order to eliminate the possible PCR-induced env recombinants, we used Phusion High-Fidelity DNA Polymerase (NEB) which can rapidly complete the DNA extension (15-30 s per $\mathrm{Kb}$ ) and longer extension time $(3 \mathrm{~min})$ than the requested $1.3 \mathrm{~min}$. PCR products $(\sim 2.6 \mathrm{~kb})$ of HIV-1 recombinant envs were gel purified with a QIAquick gel extraction kit (Qiagen), and were cloned into TOPO-TA PCR cloning vector (Invitrogen) which was then transformed into OneShot Top10 E. coli cells (Invitrogen) according to manufacturer's instructions. The transformed cells were plated onto LB (LuriaBertani) plates containing $100 \mu \mathrm{g} / \mathrm{ml}$ ampicillin, X-gal and Isopropyl $\beta$-D-1-thiogalactopyranoside (IPTG). The yielded clones were grown for plasmid extraction using a QIAamp plasmid miniprep kit (Qiagen) and the resultant DNA was sent for clonal sequencing. Full-length and V1V5 domains of recombinant gp120 were amplified from TOPO vectors using sense primer VPU-A-1, and different antisense primers gp120-D-2 (nt7617 to nt7642, 5'CCTCCTCCAGGTCTGAAGGTTTCATT-3') and V5D-2 (nt7373 to nt7399, 5'-TTAAACATATGTGTTG TAATCTCTAA-3') respectively. To further exclude the possible recombinants generated by the Taq polymerase jumping between the templates, we set up a control PCR by using the mixed proviral DNAs from the monoinfections with subtype A or D isolates. The results showed that the frequency of these background recombinants was always lower than $0.5 \%$, or at least 20 - fold less than the HIV-1 recombination frequency obtained with heterozygous virions.

\section{Cloning of full length recombinant envs and gp120s into NL4-3 backbone}

The pREC_nfl_HIV ${ }_{\text {NL4-3 }}$ vector containing a near full length (nfl) HIV-1 isolate NL4-3 backbone has previously 
been described [34] and routinely used to clone different HIV genes to create various chimeric HIV-1 viruses through yeast gap repair homologous recombination $[2$, $65,66]$. To clone an HIV-1 sequence into pREC_nfl_HIV $\mathrm{NL4-3}_{\text {- }}$ vector, the corresponding region in the NL4-3 backbone is first replaced with the yeast orotidine 5-phosphate decarboxylase (URA3) gene using specific primers tagged with 40-60 nucleotides to allow for homologous recombination at either side of the target sequence. We created cloning shuttle plasmids pREC_nfl_HIVAenv/URA3, pREC_nfl_HIVAgp120/URA3, pREC_nfl_ HIV $\Delta$ V1-V5/URA3 and pREC_nfl_HIV envA/D $_{\text {Dtat1/URA3 }}$ for cloning full length recombinant HIV-1 env, gp120, gp120 V1-V5 domains and D109 tat1 respectively. To clone the URA3 products into the NL4-3 backbone to create the corresponding shuttle plasmids, The Saccharomyces Cerevisiae yeast MYA-906 (genotype MAT $\alpha$-ade6-can1-his3leu2-trp1-URA3) was grown in $5 \mathrm{ml} \mathrm{YEPD} \mathrm{media} \mathrm{at} 30{ }^{\circ} \mathrm{C}$ on a shaking platform overnight. After $12-16 \mathrm{~h}, 0.5 \mathrm{ml}$ of the yeast culture was inoculated in $50 \mathrm{ml}$ YEPD media and grown for 4-6 h to attain an OD600 of 1.0. The yeast was washed in $1 \mathrm{ml}$ of sterile water and resuspended in $1 \mathrm{ml}$ of fresh $1 \times \mathrm{LiAc} / \mathrm{TE}$ solution in water [LiAc (100 mM pH 7.5) and TE (10 mM Tris-Cl pH 7.5 and $1 \mathrm{mM}$ EDTA $\mathrm{pH}$ 8.0)] to induce competence and then chilled on ice. The PCR inserts $(3 \mu \mathrm{g})$ and the linearized pREC_nfl_HIV $V_{\text {NL4-3 }}$ plasmid $(1 \mu g)$ were then cotransformed along with $50 \mu \mathrm{g}$ of denatured salmon sperm carrier DNA (BD Biosciences/Clontech, Palo Alto, CA) into yeast using the lithium acetate-PEG. The mixture was incubated on a shaking platform at $30{ }^{\circ} \mathrm{C}$ for $30 \mathrm{~min}$ followed by a $15 \mathrm{~min}$ heat shock at $42{ }^{\circ} \mathrm{C}$ and then plated on complete supplement media (CSM)LEU-URA agar plates in which only the yeast containing successful replacement of certain HIV sequences (i.e. env, gp120,V1-V5, or tat1/env) by URA3 gene can grow. Plasmids were retrieved from yeast clones using a mixture of mechanical glass bead disruption and phenol-chloroform-IAA extraction, and $4 \mu \mathrm{L}$ of the crude yeast preparation was transformed into STBL4 E.coli (Invitrogen). The shuttle plasmids pREC_nfl_HIVAenv/URA3, pREC_nfl_HIVAgp120/URA3, pREC_ nfl_HIV $\Delta$ V1-V5/URA3 and pREC_nfl_HIV ${ }_{\text {envA/D }} \Delta$ tat $1 /$ URA3 were finally extracted from STB14 E. coli and were used for the subsequent experimentation.

To clone the different PCR products into their corresponding shuttle plasmids, the same yeast transformation procedure was performed, but with different shuttle vectors and selected on CMM-Leu + 5-fluoro-1,2,3,6-tetrahydro2,6-dioxo-4-pyrimidine carboxylic acid (5-FOA) plates in which only the yeast containing the shuttle vector with successful replacement of URA3 by the corresponding HIV-1 env (or gp120, V1-V5, and tat1/env) recombinants can survive and generate pREC_nfl_HIVenvA/D,
pREC_nfl_HIVgp120A/D, pREC_nfl_HIV_V1-V5A/D and pREC_nfl_HIVtat1/envA/D.

\section{Veritrop assay}

One million U87.CD4.CCR5 cells were plated in $100 \mathrm{~mm}$ petri dishes (Day 1), and 24 h later (Day 2), were transfected with pDM.128-fLuc plasmid using FuGENE 6 transfection reagent (Roche). On day 1 also, $6.5 \times 10^{4}$ HEK-293 T cells (per well) were plated in 24-well plate, and $24 \mathrm{~h}$ later (Day 2), were transfected with pREC_nfl_HIV plasmid containing the respective env recombinant inserts. Six hours post-transfection, U87.CD4.CCR5 cells were plated into a 24-well plate at $6.5 \times 10^{4}$ cells per well. On day 3 , transfected HEK$293 \mathrm{~T}$ cells from each well were collected and $6.5 \times 10^{4}$ cells were layered over the seated 87.CD4.CCR5 cells and co-cultured for an additional $15 \mathrm{~h}$. Lastly, the cells were lysed with $100 \mu \mathrm{l}$ of Glo Lysis Buffer for $15 \mathrm{~min}$ at room temperature, and then $50 \mu \mathrm{l}$ of lysate was mixed with $50 \mu \mathrm{l}$ of Bright Glo (Promega Biotech) and luminescence was read on Victor ${ }^{3} \mathrm{~V}$ (Perkin Elmer) luminometer for luciferase activity.

\section{Virus production}

Production of infectious HIV-1 from pREC_nfl_HIVenvA/D (or pREC_nfl_HIVgp120A/D, pREC_nfl_ HIV_V1-V5A/D, and pREC_nfl_HIVtat1/envA/D) vectors containing various A/D env recombinants were done according to Dudley et al. [34]. Briefly, on day 1, $7 \times 10^{4}$ HEK-293 $\mathrm{T}$ cells/well were plated, and $24 \mathrm{~h}$ later (Day 2), $0.3 \mu \mathrm{g}$ of each of the pREC_nfl_HIVenvA/D plasmids containing various HIV-1 A/D env recombinant sequences were co-transfected with an equal amount of a complementing plasmid pREC_5 LTR_gag/pol (containing only the R, U5, PBS, uncoding regions, gag, and partial pol sequences) [67]. The next day (day 3) $7 \times 10^{4}$ U87.CD4.CCR5 cells were plated in a 24 well plate, and infected $24 \mathrm{~h}$ later (Day 4) by transfer of the supernatant from the transfected HEK-293 T cells. After 24 h, infection supernatant was washed off and the U87.CD4.CCR5 cell cultures maintained for up to 2 weeks following infection. Virus replications in U87.CD4.CCR5 cell culture were monitored by visually monitoring syncytia appearance and measuring reverse transcriptase activity every 2-3 days. Supernatant from infected wells was harvested and frozen at $-80^{\circ} \mathrm{C}$ for future use.

\section{Reverse transcriptase-activity assay (RT Assay)}

Ten microliter of cell culture supernatant was collected into a 96-well plate every other day and subjected to RT activity assay as previously described [68]. Briefly, $10 \mu \mathrm{l}$ of transfected and/or infected supernatant was incubated at $37{ }^{\circ} \mathrm{C}$ overnight in the presence of $25 \mu \mathrm{l}$ T mixture 
(1 $\mu \mathrm{l}$ of fresh $10 \mathrm{mCi} / \mathrm{mL}[\alpha-32 \mathrm{P}]-\mathrm{dTTP}$ in $1 \mathrm{ml} \mathrm{RT}$ master mix: $50 \mathrm{mM}$ Tris- $\mathrm{HCl}(\mathrm{pH} 7.8), 75 \mathrm{mM} \mathrm{KCl}$, $2 \mathrm{mM}$ DTT, $5 \mathrm{mM} \mathrm{MgCl} 2,5 \mu \mathrm{g} / \mathrm{ml}$ of poly(rA), $6.25 \mu \mathrm{g} /$ $\mathrm{ml}$ oligo(dT), and $0.5 \%(\mathrm{v} / \mathrm{v}) \mathrm{NP} 40)$. After incubation, $10 \mu \mathrm{l}$ of the reaction mixture was blotted from each well onto the 96-well format DEAE filtermat. After $10 \mathrm{~min}$ of drying, the filtermat was washed five times with $1 \times$ SSC solution and twice with $85 \%$ ethanol $(5 \mathrm{~min}$ for each time) in a shaking platform. The filters were then wrapped with Saran wrap and either exposed overnight onto autoradiography film (Kodak BioMax MR) or counted with a Matrix $96 \beta$-counter (Packard, Meriden, CT) to acquire the quantitative RT activity.

\section{Clonal sequencing}

We procured commercial sequencing services of ACGT, Inc (Wheeling, Illinois). Full length envs were sequenced using five universal primers Vpu-1 (nt6108-6130; sense, 5' TAATAATAGCAATAGTTGTGTGG-3'), E80 (nt68626883; sense, 5'-TTCCAATACACTATTGTGCTCC-3'), EAD2 (nt8064-8084; antisense, 5'-CCAGAGATTTAT TACTCCA-3'), E15 (nt8424-8425; antisense, 5'-CTTG CTCTCCACCTTCTTCTTC-3') and ENVM (nt90689096; antisense, 5' -TAGCCCTTCCAGTCCCCCCTTTTC TTTTA-3'). Sequencing chromatographs were physically inspected to ensure accuracy of sequencing data, and fragments were joined to form full length envs and aligned along with primary envs of isolates A91, A115, and D109 with BioEdit Sequence analysis software. Aligned sequences were imported into SimPlot sequence similarity plotting software and bootstrap plots were constructed with the recombinant sequences as the query and the two recombining primary envs as the references. The Kimura-2 parameter model was used for analysis with a 200 and 20 base pair window and step respectively.

\section{In-house ELISA}

Costar 96-well EIA/RIA plates (Immunochemistry technologies) were coated with $100 \mathrm{ng} /$ well of concentrated pseudoviruses or with commercially obtained recombinant HIV-1 gp140 (UG37) (as a positive control). Detection of envelope expression on pseudoviruses was done using of b13 monoclonal antibody (provided by George Lewis, Institute of Human Virology, Baltimore, MD, and Bruce Chesebro, NIAID, Hamilton, MT), followed by addition of rabbit anti-mouse IgG-HRP conjugate antibody (Promega Cat\#W4021) at a dilution of 1:2500, and TMB substrate. The reaction was stopped with $1 \mathrm{~N}$ sulfuric acid, the developed chromogen was read at $\lambda=$ $450 \mathrm{~nm}$, and the optical density (OD) was plotted.

\section{Western blot}

HIV-1 viral Env proteins were detected by Western blot. Blots were performed on virus pelleted from the culture supernatants. Virus-containing supernatants were clarified of cellular debris by centrifugation $(2500 \mathrm{rpm}$, $15 \mathrm{~min}$ ) and virions were pelleted by centrifugation at $32,000 \mathrm{rpm}$ for $1 \mathrm{~h}$. Samples were resuspended in sodium dodecyl sulfate (SDS) lysis buffer (40 mM Tris$\mathrm{HCl}$ [pH 6.8], $10 \%$ glycerol, $10 \% \beta$-mercaptoethanol, $1 \%$ SDS) and heated to $95{ }^{\circ} \mathrm{C}$ for $5 \mathrm{~min}$, separated by SDS-10 \% polyacrylamide gel electrophoresis, and transferred to nitrocellulose. Membranes were blocked with $5 \%$ milk, incubated with the B13 antibody and then with horseradish peroxidase-conjugated secondary antibodies, detected with the ECL Plus kit (Amersham Biosciences, Piscataway, NJ), and finally exposed to $\mathrm{x}$ ray film.

\section{Phylogenetic/coevolution analyses}

Intra-protein coevolution may be inferred by quantifying the degree of similarity of phylogenetic tree structure of two protein domains based on the correlation of the distance matrices used to build the trees [47]. To measure the covariance between the conserved regions of gp120 and the gp41 extracellular domain, full length subtype A1 and subtype D envelope amino acid sequences were downloaded from the Los Alamos National Laboratory HIV sequence database (http://www.hiv.lanl.gov/). The sequences were filtered to include one sequence per patient and sequences with more than one ambiguous codon were removed, which resulted in data sets containing 144 and 97 unique sequences, respectively. Subtype specific alignments were performed using MUSCLE [69] then trimmed to the positions containing the $\mathrm{C} 1-$ C5 regions and the ecto-gp41 domain (Fig. 8a). Maximum likelihood trees were generated for each region using the algorithms implemented in PHYML [70] with 100 bootstrap replicates, and the tree topology search was performed using Nearest Neighbor Interchanges (NNIs). The phylogeny of the regions was estimated using the rtREV [45] model of amino acid substitution, which was developed specifically for phylogenetic inference of retroviral sequence. The equilibrium frequencies and the gamma distribution were both estimated by the model, and the number of substitution rate categories was set to 4 . The resulting maximum likelihood trees were used as input to the Mirrortree web server [46] which was developed to predict functional relationships between protein families based on the tree topology of the proteins or domains being compared (Fig. 8b). This algorithm implements the tree correlation method developed by Pazos and Valencia [47] that creates a distance matrix for each tree by summing the branch lengths that separate the taxa in the tree, and then estimating the linear correlation coefficient $(r)$ between the two matrices. Heatmaps demonstrating the correlation 
between each region in env were produced with Plot (https://plot.ly).

Additionally, pairwise distance matrices of each region were built with MEGA [71], which used a Jones-TaylorThornton (JTT) matrix-based model [48] and a uniform substitution rate among sites, to calculate the number of amino acid substitutions per site/residue from between each sequence in the alignment. Correlation between each conserved region in gp120 or the gp41 domain were then measured by Pearson's product-moment correlation coefficient, and significance estimated with Fisher's exact test (Fig. 8c).

\section{Abbreviations \\ HIV-1: Human immunodeficiency virus type 1; URF: Unique recombinant form; CRF: Circulating recombinant form.}

\section{Competing interests}

The authors declare that they have no competing interests.

\section{Authors' contributions}

BSB designed the study, performed the experiments and drafted the manuscript. JFV, MT, YL and KCK performed various vector construction and virus generation. GN performed the analysis of co-evolution of $\mathrm{Cl}$ and $\mathrm{C5}$ / gp41. EJA provided some suggestions for the project and helped the writing of the manuscript. YG provided overall supervision for the project, secured funding, and helped the writing the manuscript. All authors read and approved the final manuscript.

\section{Acknowledgements}

This study was supported by research grants awarded to Y.G. and E.J.A., NIH/ NIAID Al49170 and Al84816, and by the Fogarty International Center through NIH R21 Al079852.

\section{Author details}

'Department of Molecular Biology and Microbiology, School of Medicine, Case Western Reserve University, 10900 Euclid Ave, Cleveland, OH 44106, USA. ${ }^{2}$ Division of Infectious Diseases, Department of Medicine, Case Western Reserve University, 10900 Euclid Ave, Cleveland, OH 44106, USA.

${ }^{3}$ Department of Microbiology and Immunology, Schulich School of Medicine and Dentistry, Western University, London, ON N6A 5C1, Canada.

Received: 30 March 2015 Accepted: 2 April 2015

Published online: 23 May 2015

\section{References}

1. Baird HA, Gao Y, Galetto R, Lalonde M, Anthony RM, Giacomoni V, et al. Influence of sequence identity and unique breakpoints on the frequency of intersubtype HIV-1 recombination. Retrovirology. 2006;3:91.

2. Gao Y, Abreha M, Nelson KN, Baird H, Dudley DM, Abraha A, et al. Enrichment of intersubtype HIV-1 recombinants in a dual infection system using HIV-1 strain-specific siRNAs. Retrovirology. 2011;8:5.

3. Hemelaar J. Implications of HIV diversity for the HIV-1 pandemic. J Infect. 2013:66:391-400

4. Keele BF, Derdeyn CA. Genetic and antigenic features of the transmitted virus. Curr Opin HIV AIDS. 2009;4:352-7.

5. Lu S, Grimes Serrano JM, Wang S. Polyvalent AIDS vaccines. Curr HIV Res. 2010:8:622-9.

6. Santos LA, Monteiro-Cunha JP, Araujo AF, Brites C, Galvao-Castro B, Alcantara LC. Detection of distinct human immunodeficiency virus type 1 circulating recombinant forms in northeast Brazil. J Med Virol. 2011:83:2066-72.

7. Feng $Y$, Wei $H, H s i J$, Xing H, He X, Liao L, et al. Identification of a novel HIV-1 circulating recombinant form (CRF65_cpx) comprised of CRF01_AE and subtypes B and C in western Yunnan. China: AIDS Res Hum Retroviruses; 2013.

8. Fernandez-Garcia A, Perez-Alvarez L, Cuevas MT, Delgado E, Munoz-Nieto M, Cilla G, et al. Identification of a new HIV type 1 circulating BF intersubtype recombinant form (CRF47_BF) in Spain. AIDS Res Hum Retroviruses. 2010;26:827-32.
9. Han X, An M, Zhang W, Zhao B, Chu Z, Takebe Y, et al. Genome Sequences of a Novel HIV-1 Circulating Recombinant Form (CRF59_01B) Identified among Men Who Have Sex with Men in Northeastern China. Genome Announc. 2013;1(3):e00315-13.

10. Monno L, Brindicci G, Lai A, Punzi G, Altamura M, Simonetti FR, et al. An outbreak of HIV-1 BC recombinants in Southern Italy. J Clin Virol. 2012;55:370-3.

11. Ng KT, Ong LY, Takebe Y, Kamarulzaman A, Tee KK. Genome sequence of a novel HIV-1 circulating recombinant form 54_01B from Malaysia. J Virol. 2012;86:11405-6.

12. Ong LY, Razak SN, Lee YM, Sri Sr L, Syed Omar SF, Azwa Rl, et al. Molecular diversity of HIV-1 and surveillance of transmitted drug resistance variants among treatment Naive patients, 5 years after active introduction of HAART in Kuala Lumpur, Malaysia. J Med Virol. 2014;86:38-44.

13. Hemelaar J, Gouws E, Ghys PD, Osmanov S. Global trends in molecular epidemiology of HIV-1 during 2000-2007. AIDS. 2011;25:679-89.

14. Tatem AJ, Hemelaar J, Gray RR, Salemi M. Spatial accessibility and the spread of HIV-1 subtypes and recombinants. AIDS. 2012;26:2351-60.

15. Kemal KS, Ramirez CM, Burger H, Foley B, Mayers D, Klimkait T, et al. Recombination between variants from genital tract and plasma: evolution of multidrug-resistant HIV type 1. AIDS Res Hum Retroviruses. 2012;28:1766-74.

16. Quan $Y$, Liang C, Brenner BG, Wainberg MA. Multidrug-resistant variants of HIV type 1 (HIV-1) can exist in cells as defective quasispecies and be rescued by superinfection with other defective HIV-1 variants. J Infect Dis. 2009;200:1479-83.

17. Simon-Loriere E, Rossolillo P, Negroni M. RNA structures, genomic organization and selection of recombinant HIV. RNA Biol. 2011;8:280-6.

18. Neher RA, Leitner T. Recombination rate and selection strength in HIV intra-patient evolution. PLoS Comput Biol. 2010;6:e1000660.

19. Bull JJ, Sanjuan R, Wilke CO. Theory of lethal mutagenesis for viruses. J Virol. 2007:81:2930-9.

20. Haim H, Salas I, Sodroski J. Proteolytic processing of the human immunodeficiency virus envelope glycoprotein precursor decreases conformational flexibility. J Virol. 2013;87:1884-9.

21. Moulard M, Hallenberger S, Garten W, Klenk HD. Processing and routage of HIV glycoproteins by furin to the cell surface. Virus Res. 1999;60:55-65.

22. Freed EO. HIV-1 replication. Somat Cell Mol Genet. 2001;26:13-33.

23. Moore JP, Willey RL, Lewis GK, Robinson J, Sodroski J. Immunological evidence for interactions between the first, second, and fifth conserved domains of the gp120 surface glycoprotein of human immunodeficiency virus type 1. J Virol. 1994;68:6836-47.

24. Shang L, Yue L, Hunter E. Role of the membrane-spanning domain of human immunodeficiency virus type 1 envelope glycoprotein in cell-cell fusion and virus infection. J Virol. 2008;82:5417-28.

25. Shang $L$, Hunter E. Residues in the membrane-spanning domain core modulate conformation and fusogenicity of the HIV-1 envelope glycoprotein. Virology. 2010;404:158-67.

26. Purcell DF, Martin MA. Alternative splicing of human immunodeficiency virus type 1 mRNA modulates viral protein expression, replication, and infectivity. J Virol. 1993:67:6365-78.

27. Tazi J, Bakkour N, Marchand V, Ayadi L, Aboufirassi A, Branlant C. Alternative splicing: regulation of HIV-1 multiplication as a target for therapeutic action. FEBS J. 2010;277:867-76.

28. Rossenkhan R, Novitsky V, Sebunya TK, Musonda R, Gashe BA, Essex M. Viral diversity and diversification of major non-structural genes vif, vpr, vpu, tat exon 1 and rev exon 1 during primary HIV-1 subtype C infection. PLoS One. 2012; $7:$ e35491.

29. Rossenkhan R, MacLeod IJ, Sebunya TK, Castro-Nallar E, McLane MF Musonda R, et al. tat Exon 1 exhibits functional diversity during HIV-1 subtype C primary infection. J Virol. 2013;87:5732-45.

30. Baird HA, Galetto R, Gao Y, Simon-Loriere E, Abreha M, Archer J, et al. Sequence determinants of breakpoint location during HIV-1 intersubtype recombination. Nucleic Acids Res. 2006;34:5203-16.

31. Quinones-Mateu ME, Gao Y, Ball SC, Marozsan AJ, Abraha A, Arts EJ. In vitro intersubtype recombinants of human immunodeficiency virus type 1: comparison to recent and circulating in vivo recombinant forms. J Virol. 2002:76:9600-13

32. Quinones-Mateu ME, Ball SC, Marozsan AJ, Torre VS, Albright JL, Vanham G, et al. A dual infection/competition assay shows a correlation between ex vivo human immunodeficiency virus type 1 fitness and disease progression. J Virol. 2000;74:9222-33. 
33. Archer J, Pinney JW, Fan J, Simon-Loriere E, Arts EJ, Negroni M, et al. Identifying the important HIV-1 recombination breakpoints. PLoS Comput Biol. 2008:4:e1000178.

34. Dudley DM, Gao Y, Nelson KN, Henry KR, Nankya I, Gibson RM, et al. A novel yeast-based recombination method to clone and propagate diverse HIV-1 isolates. Biotechniques. 2009;46:458-67.

35. Weber J, Vazquez AC, Winner D, Gibson RM, Rhea AM, Rose JD, et al. Sensitive cell-based assay for determination of human immunodeficiency virus type 1 coreceptor tropism. J Clin Microbiol. 2013;51:1517-27.

36. Bartesaghi A, Merk A, Borgnia MJ, Milne JL, Subramaniam S. Prefusion structure of trimeric HIV-1 envelope glycoprotein determined by cryo-electron microscopy. Nat Struct Mol Biol. 2013;20:1352-7.

37. Julien JP, Cupo A, Sok D, Stanfield RL, Lyumkis D, Deller MC, et al. Crystal structure of a soluble cleaved HIV-1 envelope trimer. Science. 2013;342:1477-83.

38. Lyumkis D, Julien JP, De VN, Cupo A, Potter CS, Klasse PJ, et al. Cryo-EM structure of a fully glycosylated soluble cleaved HIV-1 envelope trimer. Science. 2013;342:1484-90.

39. Galli A, Kearney M, Nikolaitchik OA, Yu S, Chin MP, Maldarelli F, et al. Patterns of Human Immunodeficiency Virus type 1 recombination ex vivo provide evidence for coadaptation of distant sites, resulting in purifying selection for intersubtype recombinants during replication. J Virol. 2010:84:7651-61.

40. Binley JM, Sanders RW, Clas B, Schuelke N, Master A, Guo Y, et al. A recombinant human immunodeficiency virus type 1 envelope glycoprotein complex stabilized by an intermolecular disulfide bond between the gp120 and gp41 subunits is an antigenic mimic of the trimeric virion-associated structure. J Virol. 2000:74:627-43.

41. Poumbourios P, Maerz AL, Drummer HE. Functional evolution of the HIV-1 envelope glycoprotein 120 association site of glycoprotein 41. J Biol Chem. 2003;278:42149-60.

42. Sen J, Jacobs A, Caffrey M. Role of the HIV gp120 conserved domain 5 in processing and viral entry. Biochemistry. 2008;47:7788-95.

43. Sen J, Yan T, Wang J, Rong L, Tao L, Caffrey M. Alanine scanning mutagenesis of HIV-1 gp41 heptad repeat 1: insight into the gp120-gp41 interaction. Biochemistry. 2010;49:5057-65.

44. Wang J, Sen J, Rong L, Caffrey M. Role of the HIV gp120 conserved domain 1 in processing and viral entry. J Biol Chem. 2008:283:32644-9.

45. Dimmic MW, Rest JS, Mindell DP, Goldstein RA. rtREV: an amino acid substitution matrix for inference of retrovirus and reverse transcriptase phylogeny. J Mol Evol. 2002;55:65-73.

46. Ochoa D, Pazos F. Studying the co-evolution of protein families with the Mirrortree web server. Bioinformatics. 2010;26:1370-1.

47. Pazos F, Valencia A. Similarity of phylogenetic trees as indicator of proteinprotein interaction. Protein Eng. 2001;14:609-14.

48. Jones DT, Taylor WR, Thornton JM. The rapid generation of mutation data matrices from protein sequences. Comput Appl Biosci. 1992;8:275-82.

49. Powell RL, Lezeau L, Kinge T, Nyambi PN. Longitudinal quasispecies analysis of viral variants in HIV type 1 dually infected individuals highlights the importance of sequence identity in viral recombination. AIDS Res Hum Retroviruses, 2010:26:253-64

50. Rhodes TD, Nikolaitchik O, Chen J, Powell D, Hu WS. Genetic recombination of human immunodeficiency virus type 1 in one round of viral replication: effects of genetic distance, target cells, accessory genes, and lack of high negative interference in crossover events. J Virol. 2005;79:1666-77.

51. Woo J, Robertson DL, Lovell SC. Constraints from protein structure and intra-molecular coevolution influence the fitness of HIV-1 recombinants. Virology. 2014;454-455:34-9.

52. Chen B, Vogan EM, Gong H, Skehel JJ, Wiley DC, Harrison SC. Structure of an unliganded simian immunodeficiency virus gp120 core. Nature. 2005:433:834-41.

53. Huang CC, Tang M, Zhang MY, Majeed S, Montabana E, Stanfield RL, et al. Structure of a V3-containing HIV-1 gp120 core. Science. 2005;310:1025-8.

54. Kwong PD, Wyatt R, Robinson J, Sweet RW, Sodroski J, Hendrickson WA Structure of an HIV gp120 envelope glycoprotein in complex with the CD4 receptor and a neutralizing human antibody. Nature. 1998:393:648-59.

55. Wyatt R, Desjardin E, Olshevsky U, Nixon C, Binley J, Olshevsky V, et al. Analysis of the interaction of the human immunodeficiency virus type 1 gp120 envelope glycoprotein with the gp41 transmembrane glycoprotein. J Virol. 1997:71:9722-31.
56. Pancera M, Majeed S, Ban YE, Chen L, Huang CC, Kong L, et al. Structure of HIV-1 gp120 with gp41-interactive region reveals layered envelope architecture and basis of conformational mobility. Proc Natl Acad Sci U S A. 2010;107:1166-71.

57. Moore JP, McKeating JA, Weiss RA, Sattentau QJ. Dissociation of gp120 from HIV-1 virions induced by soluble CD4. Science. 1990;250:1139-42.

58. Agrawal N, Leaman DP, Rowcliffe E, Kinkead H, Nohria R, Akagi J, et al. Functional stability of unliganded envelope glycoprotein spikes among isolates of human immunodeficiency virus type 1 (HIV-1). PLoS One. 2011;6:e21339.

59. Moore JP, Klasse PJ. Thermodynamic and kinetic analysis of sCD4 binding to HIV-1 virions and of gp120 dissociation. AIDS Res Hum Retroviruses. 1992:8:443-50

60. Simon-Loriere E, Galetto R, Hamoudi M, Archer J, Lefeuvre P, Martin DP, et al. Molecular mechanisms of recombination restriction in the envelope gene of the human immunodeficiency virus. PLoS Pathog. 2009;5:e1000418.

61. Helseth $\mathrm{E}$, Olshevsky U, Furman C, Sodroski J. Human immunodeficiency virus type 1 gp120 envelope glycoprotein regions important for association with the gp41 transmembrane glycoprotein. J Virol. 1991;65:2119-23.

62. Jacobs A, Sen J, Rong L, Caffrey M. Alanine scanning mutants of the HIV gp41 loop. J Biol Chem. 2005;280:27284-8.

63. Gao Y, Paxinos E, Galovich J, Troyer R, Baird H, Abreha M, et al. Characterization of a subtype D human immunodeficiency virus type 1 isolate that was obtained from an untreated individual and that is highly resistant to nonnucleoside reverse transcriptase inhibitors. J Virol. 2004;78:5390-401.

64. Domingo $E_{1}$ Holland JJ. RNA virus mutations and fitness for survival. Annu Rev Microbiol. 1997;51:151-78.

65. Lobritz MA, Ratcliff AN, Marozsan AJ, Dudley DM, Tilton JC, Arts EJ. Multifaceted mechanisms of HIV inhibition and resistance to CCR5 inhibitors PSC-RANTES and Maraviroc. Antimicrob Agents Chemother. 2013:57:2640-50.

66. Ratcliff AN, Shi W, Arts EJ. HIV-1 resistance to maraviroc conferred by a CD4 binding site mutation in the envelope glycoprotein gp120. J Virol. 2013:87:923-34.

67. Chamanian M, Purzycka KJ, Wille PT, Ha JS, McDonald D, Gao Y, et al. A cis-acting element in retroviral genomic RNA links Gag-Pol ribosomal frameshifting to selective viral RNA encapsidation. Cell Host Microbe. 2013;13:181-92.

68. Ball SC, Abraha A, Collins KR, Marozsan AJ, Baird H, Quinones-Mateu ME, et al. Comparing the ex vivo fitness of CCR5-tropic human immunodeficiency virus type 1 isolates of subtypes B and C. J Virol. 2003;77:1021-38.

69. Edgar RC. MUSCLE: multiple sequence alignment with high accuracy and high throughput. Nucleic Acids Res. 2004;32:1792-7.

70. Guindon S, Dufayard JF, Lefort V, Anisimova M, Hordijk W, Gascuel O. New algorithms and methods to estimate maximum-likelihood phylogenies: assessing the performance of PhyML 3.0. Syst Biol. 2010;59:307-21.

71. Tamura K, Stecher G, Peterson D, Filipski A, Kumar S. MEGA6: molecular evolutionary genetics analysis version 6.0. Mol Biol Evol. 2013;30:2725-9.

\section{Submit your next manuscript to BioMed Central and take full advantage of:}

- Convenient online submission

- Thorough peer review

- No space constraints or color figure charges

- Immediate publication on acceptance

- Inclusion in PubMed, CAS, Scopus and Google Scholar

- Research which is freely available for redistribution 